\title{
A MULTILEVEL PRECONDITIONER FOR THE INTERIOR PENALTY DISCONTINUOUS GALERKIN METHOD*
}

\author{
KOLJA BRIX ${ }^{\dagger}$, MARTIN CAMPOS PINTO ${ }^{\ddagger}$, AND WOLFGANG DAHMEN $§$
}

\begin{abstract}
In this article we present a multilevel preconditioner for interior penalty discontinuous Galerkin discretizations of second order elliptic boundary value problems that gives rise to uniformly bounded condition numbers without any additional regularity assumptions on the solution. The underlying triangulations are only assumed to be shape regular but may have hanging nodes subject to certain mild grading conditions. A key role is played by certain decompositions of the discontinuous trial space into a conforming subspace and a non-conforming subspace that is controlled by the jumps across the edges.
\end{abstract}

AMS Subject Classification: 65F10, 65N55, 65N30

Key Words: Interior penalty method, energy-stable splittings, admissible averaging operators, frames, multilevel Schwarz preconditioners, discontinuous Galerkin methods.

1. Introduction. An attractive feature of discontinuous Galerkin (DG) discretizations is their suitability for a variety of different problem types as well as the flexibility regarding local mesh refinement and even locally varying the order of the discretization. While initially the main focus has been on transport problems like hyperbolic conservation laws interest has shifted recently towards diffusion problems. One simple reason is that such problems naturally enter the picture in more complex applications like the compressible or incompressible Navier-Stokes equations. By now numerous papers have been devoted to well-posedness and stability issues as well as to the derivation of (a priori and a posteriori) error estimation 2, 1, 10, 12. In comparison much less seems to be known about efficient solution methods for the resulting systems of equations. An important step in this direction has been 8 which proposes a multigrid scheme (covering actually convection diffusion problems as well) that does give rise to uniformly bounded condition numbers for diffusive problems provided that (i) the underlying hierarchy of meshes is quasi-uniform and (ii) the solution exhibits a certain (weak) regularity.

On the other hand, the local nature of the method suggests a particular suitability for problems with singular behavior calling for inhomogeneous meshes and possibly weak regularity requirements. Therefore the main objective of this paper is to present a preconditioner that is optimal in the sense of providing uniformly bounded condition numbers even for meshes that are shape regular but only locally quasiuniform and have hanging nodes, arising e.g. from local mesh refinements. Moreover, no extra regularity assumptions are needed. The approach is quite different from [8] and uses stable splittings and concepts from the theory of multilevel Schwarz schemes.

As one important by-product of our analysis, we prove that a simple splitting of any discontinuous, high order piecewise polynomial space $V_{h}:=\mathbb{P}_{k}\left(\mathcal{T}_{h}\right)$ based on its first degree conforming subspace (the minimal conforming subspace) is stable with respect to the standard mesh dependent norm $\|\cdot\|_{h}$ associated with the DG discretization of an $H^{1}$-elliptic boundary value problem (see 2.77). The essential (and necessary) requirement for this is that the triangulation $\mathcal{T}_{h}$ underlying $V_{h}$, that may have hanging nodes due to local refinements, satisfies a certain grading property. A special and somewhat simplified version of these findings can be formulated as follows.

THEOREM 1.1. Let $\Phi_{h}:=\left\{\phi_{i}: i \in \mathcal{I}_{h}\right\}$ be a standard local basis of $V_{h}$, i.e. each basis function is a piecewise polynomial supported in a single triangle, and let $V_{h, 1}^{\mathrm{c}}:=H_{0}^{1}(\Omega) \cap \mathbb{P}_{1}\left(\mathcal{T}_{h}\right) \subset V_{h}$ denote the space

\footnotetext{
*This work has been supported in part by the French-German PROCOPE contract 11418YB, by the European Commission Human Potential Programme under contract HPRN-CT-2002-00286, "Breaking Complexity", by the SFB 401 and the Leibniz Programme funded by DFG.

${ }^{\dagger}$ Institut für Geometrie und Praktische Mathematik, RWTH Aachen, 52056 Aachen, Germany, brix@igpm.rwth-aachen.de, http://www.igpm.rwth-aachen.de/brix/

${ }_{\ddagger}^{\ddagger}$ Institut de Recherche Mathématique Avancée, CNRS - Université Louis Pasteur, 67084 Strasbourg, France, campos@math.u-strasbg.fr, http://www.ann.jussieu.fr/ campos/

\$Institut für Geometrie und Praktische Mathematik, RWTH Aachen, 52056 Aachen, Germany, dahmen@igpm.rwth-aachen.de, http://www.igpm.rwth-aachen.de/dahmen/
} 
of continuous functions that are piecewise affine on $\mathcal{T}_{h}$. Then

$$
c\|v\|_{h}^{2} \leq \inf _{\substack{v^{\mathrm{c}} \in V_{h, 1}^{\mathrm{c}}, d_{i} \in \mathbb{R} \\ v=v^{\mathrm{c}}+\sum_{i \in \mathcal{I}_{h}} d_{i} \phi_{i}}}\left\|v^{\mathrm{c}}\right\|_{1}^{2}+\sum_{i \in \mathcal{I}_{h}}\left\|d_{i} \phi_{i}\right\|_{h}^{2} \leq C\|v\|_{h}^{2}, \quad v \in V_{h}
$$

holds for constants $c$ and $C$ independent of $\mathcal{T}_{h}$, provided that the above mentioned grading property holds.

The layout of the paper is as follows. In Section 2 we set notation, formulate the model problem and recall some relevant facts about the symmetric interior penalty discontinuous Galerkin method (SIPG). In Section 3 we briefly review the relevant aspects of additive Schwarz schemes that provide the framework for our preconditioners. Specifically, in Section 3.1 we point out first that a certain naive attempt motivated by wavelet-type preconditioners does not work. This is unfortunate since so-called multiwavelets would offer a simple tool in the present context. The explanation why this fails is instructive in that it highlights the necessity of working with suitable splits of the trial spaces into a conforming part and a part that could solely be controlled by the jumps across element edges. Section 4 is devoted to the identification of suitable stable splittings that give rise to uniformly bounded condition numbers. There are essentially two crucial issues that are perhaps worth mentioning. First, the required grouping of stable splittings into conforming and nonconforming parts requires nontrivial conforming subspaces. When working with local mesh refinements with hanging vertices this will be seen to boil down to certain mild grading conditions on the meshes that are discussed in Section 4.1. Second, given such graded meshes suitable decompositions into conforming and nonconforming subspaces can be obtained through certain averaging projectors. Their essential qualifying property, namely a certain Jackson-type estimate, is developed in Section 4.2 . In fact, the main result of Section 4 is that any split induced by such an admissible projector into a conforming subspace of the whole trial space gives rise to optimal Schwarz preconditioners by simply taking the union of two stable splittings for the respective subspaces, see Theorem 1.1. Section 5 is then devoted to the detailed concrete realization of a specific admissible averaging projector whose image is the space of continuous piecewise linear finite elements. In this case stable splittings for the conforming part can be invoked from the literature while the discontinuous piecewise polynomial basis of the whole trial space turns out to be a stable splitting for the corresponding nonconforming subspace. In this case the conforming subspace is minimal. We emphasize though that this is only one possible realization among many other ones. In Section 6 we present some first numerical experiments. We conclude with indicating alternatives based on projectors onto maximal conforming subspace whose detailed analysis supported by more extensive numerical quantifications are deferred to a forthcoming paper.

We shall sometimes write $a \lesssim b$ to mean that $a$ can be bounded by some constant multiple of $b$ where the constant does not depend on any parameters on which $a, b$ may depend. Likewise $a \sim b$ means that both $a \lesssim b$ and $b \lesssim a$ hold.

2. The Symmetric Interior Penalty Method - A model problem. Although the subsequent developments carry over to higher spatial dimensions we shall confine the discussion for simplicity to the two-dimensional case. Thus, let $\Omega$ be a bounded polygonal domain in $\mathbb{R}^{2}$ and let

$$
a(u, v):=\int_{\Omega}(A \nabla u) \cdot \nabla v+\int_{\Omega} b u v
$$

where $A$ is a uniformly positive definite $2 \times 2$ matrix and $b$ is a nonnegative bounded function on $\Omega$. To avoid quadrature issues, we shall always assume that both $A$ and $b$ are piecewise constant with respect to a fixed coarse shape regular conforming triangulation $\mathcal{T}^{0}=\mathcal{T}^{0}(\Omega)$ of $\Omega$. As usual this means that the ratio of the radii of the circumscribed and inscribed circles of all triangles remains uniformly bounded. It will be convenient to work with closed triangles, i.e. the triangles $K$ will always be assumed to be closed.

We consider the following model problem: Given $f \in L_{2}(\Omega)$, find $u \in H_{0}^{1}(\Omega)$ (the usual Sobolev space of functions in $L_{2}(\Omega)$ whose first order weak derivatives belong also to $L_{2}(\Omega)$ and whose trace vanishes on $\partial \Omega$ ), such that

$$
a(u, v)=(f, v), \quad v \in H_{0}^{1}(\Omega) .
$$

The Lax-Milgram Theorem ensures the unique solvability of (2.2). In particular, we know there exist positive constants $c_{a}, C_{a}$ depending only on $\Omega, A, b$ such that for $\|v\|_{1}^{2}:=\|v\|_{L_{2}(\Omega)}^{2}+\|\nabla v\|_{L_{2}(\Omega)}^{2}$ one has

$$
c_{a}\|v\|_{1}^{2} \leq a(v, v) \quad \text { and } \quad|a(v, w)| \leq C_{a}\|v\|_{1}\|w\|_{1}, \quad v, w \in H_{0}^{1}(\Omega) .
$$


We shall discretize $(2.2)$ with a discontinuous Galerkin scheme. To describe this it will be convenient to employ the following localized inner products. For any domain $G \subseteq \Omega$ we set

$$
(v, w)_{G}:=\int_{G} v w, \quad a(v, w)_{G}:=\int_{G}(A \nabla u) \cdot \nabla v+\int_{G} b u v .
$$

2.1. The Symmetric Interior Penalty Method on locally refined triangulations . The DG discretization that we shall describe next will be based on triangulations $\mathcal{T}_{h}=\mathcal{T}_{h}(\Omega)$ that are generated from some initial shape regular conforming triangulation $\mathcal{T}^{0}$ through possibly local refinements. The subscript $h$ therefore refers to a piecewise constant mesh size function given by $h(K):=\operatorname{diam}(K)$, $K \in \mathcal{T}_{h}$. In principle, any kind of local refinement is permitted that preserves shape regularity such as partition based on "newest vertex insertion" or the standard partition of a triangle into four congruent subtriangles. One could think of closing the partitions arising from such local refinements to keep the triangulation conforming (which means any two distinct closed triangles have an empty intersection or share a common face (edge or vertex)) while preserving shape regularity. We stress though that nonconforming triangulations are permitted as well, i.e. hanging vertices (sometimes called hanging nodes) may occur, see Figure 2.1. Since the occurrence of hanging vertices does complicate the construction of the preconditioner and its analysis somewhat and since conforming shape regular triangulations may be viewed as simpler special cases we shall concentrate in the following on situations where hanging vertices occur. As usual we require the triangulations to be graded meaning that an edge may contain at most one hanging vertex, see Figure 2.1.
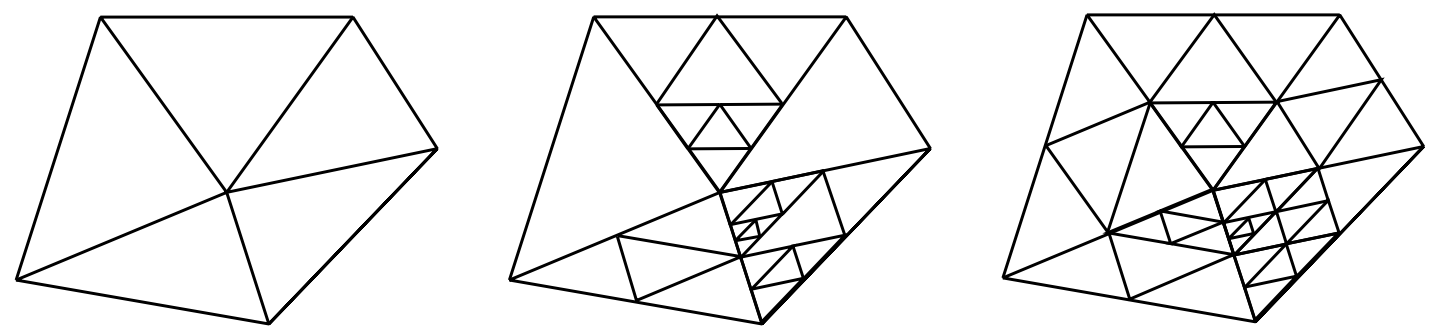

FIG. 2.1. Coarse $\mathcal{T}^{0}$ (left), refinement (middle), and graded, shape regular $\mathcal{T}_{h}$ (right)

Later we shall have to impose a slightly stronger grading property.

Denoting by $\mathbb{P}_{k}(G)$ the space of all polynomials of total degree at most $k$ over the domain $G$, we associate with any (admissible) triangulation $\mathcal{T}_{h}$ the trial space

$$
V_{h}:=\mathbb{P}_{k}\left(\mathcal{T}_{h}\right):=\bigoplus_{K \in \mathcal{T}_{h}} \mathbb{P}_{k}(K)=\left\{v=\sum_{K \in \mathcal{T}_{h}} \chi_{K} P_{K}: P_{K} \in \mathbb{P}_{k}(K)\right\}
$$

of all (possibly discontinuous) piecewise polynomials of degree at most $k$ on $\mathcal{T}_{h}$. Here $\chi_{K}$ is the standard indicator function, i.e. $\chi_{K}(x)=1$ for $x \in K$ and $\chi_{K}(x)=0$ else. The subscript $k$ is thus to be understood (in the same way as the mesh size function $h$ ) as a degree function taking a constant integer value $k=k(K)$ on each triangle $K \in \mathcal{T}_{h}$ that may vary for different $K$. However, we shall always assume that a fixed upper bound $\bar{k}$ is never exceeded, i.e. $k \leq \bar{k}$ for all $K \in \mathcal{T}_{h}$.

For the derivation of the symmetric interior penalty method we refer e.g. to [1, 2]. To formulate it in the present setting we need the following notation. Let $\mathcal{E}_{h}$ be the set of edges of the triangulation $\mathcal{T}_{h}$. Since hanging vertices are permitted we shall adopt the convention that whenever an edge has a hanging node it is replaced in $\mathcal{E}_{h}$ by its two halves. Moreover, $\mathcal{E}_{h, b}, \mathcal{E}_{h, i}$ denote the subsets of boundary and interior edges, respectively. Since triangles are always assumed to be closed sets, for any $e \in \mathcal{E}_{h, i}$ there exist $K, K^{\prime} \in \mathcal{T}_{h}$ such that $e=K \cap K^{\prime}$, and at least one of the two triangles has $e$ as an edge. It will also be convenient to use the abbreviation

$$
v^{K}:=\chi_{K} v
$$

Thus for any $v \in V_{h}$ and $K \in \mathcal{T}_{h}$ it makes sense to denote by $\left.v^{K}\right|_{e}$ the trace of the restriction of $v$ to $K$ on the subset $e$ of $\partial K$. As usual the jump of $v$ across $e$ is then defined as

$$
[v]_{e}:=\left.\mathbf{n}_{K, e} v^{K}\right|_{e}+\left.\mathbf{n}_{K^{\prime}, e} v^{K^{\prime}}\right|_{e},
$$


where $\mathbf{n}_{K, e}=-\mathbf{n}_{K^{\prime}, e}$ denotes the unit normal vector on $e$ pointing to the exterior of $K$. When $e \in \mathcal{E}_{h, b}$ is a boundary edge we think of $v$ being extended by zero outside $\Omega$. Likewise averages on $e$ are defined as

$$
\{v\}_{e}:= \begin{cases}\frac{1}{2}\left(\left.v^{K}\right|_{e}+\left.v^{K^{\prime}}\right|_{e}\right), & e \in \mathcal{E}_{h, i}, \\ \left.v^{K}\right|_{e}, & e \in \mathcal{E}_{h, b}\end{cases}
$$

(and we shall drop the subscript $e$ when it is obvious from the context). Given $\mathcal{T}_{h}$, consider the bilinear form

$$
a_{h}(v, w):=\sum_{K \in \mathcal{T}_{h}} a(v, w)_{K}-\sum_{e \in \mathcal{E}_{h}} \int_{e}(\{\nabla w\} \cdot[v]+\{\nabla v\} \cdot[w])+\sum_{e \in \mathcal{E}_{h}} \frac{\gamma}{|e|} \int_{e}[w] \cdot[v] .
$$

Here $\gamma$ is a positive (possibly piecewise) constant that has to be chosen appropriately to ensure stability of the method, see relation (2.7) below. Its existence is usually argued under slightly different assumptions on the underlying meshes but the argument easily carries over.

In fact, the only points to be addressed are perhaps the occurrence of a hanging vertex and a possible local variation of $\gamma$ depending on the coefficients in 2.1. Applying a Cauchy-Schwarz inequality to 2.4, one gets

$$
a_{h}(v, v) \geq \sum_{K \in \mathcal{T}_{h}} a(v, v)_{K}-2\left(\sum_{e \in \mathcal{E}_{h}} \frac{|e|}{\sigma}\left\|\left\{\partial_{n} v\right\}\right\|_{L_{2}(e)}^{2}\right)^{1 / 2}\left(\sum_{e \in \mathcal{E}_{h}} \frac{\sigma}{|e|}\|[v]\|_{L_{2}(e)}^{2}\right)^{1 / 2}+\sum_{e \in \mathcal{E}_{h}} \frac{\gamma}{|e|}\|[v]\|_{L_{2}(e)}^{2} .
$$

When $e=K \cap K^{\prime}$ is an edge of $K^{\prime}$ but only half of an edge of $K$, as in Figure 4.1 we can write for $v \in V_{h}$ the restriction $v^{K}$ as $\sum_{J \in \boldsymbol{C}(K)} v^{J}$. Hence, denoting by $\lambda_{\max / \min }(A)$ the maximal/minimal eigenvalue of $A$, and defining for $\omega \subseteq \Omega$

$$
\underline{\lambda}_{\omega}:=\inf _{x \in \omega} \lambda_{\min }(A(x)), \quad \bar{\lambda}_{\omega}:=\sup _{x \in \omega} \lambda_{\max }(A(x)),
$$

the usual combination of a trace inequality with an inverse inequality yields

$$
|e|\left\|\left\{\partial_{n} v\right\}\right\|_{L_{2}(e)}^{2} \leq C\left(\|\nabla v\|_{L_{2}\left(K^{\prime}\right)}^{2}+\|\nabla v\|_{L_{2}(J)}^{2}\right) \leq C \max _{J^{\prime}=K^{\prime}, J} \underline{\lambda}_{J^{\prime}}^{-1} a(v, v)_{K^{\prime} \cup J},
$$

where $J \subset K$ is the child of $K$ adjacent to $K^{\prime}$ and the constant $C$ depends on the shape properties of $\mathcal{T}^{0}$ and $\bar{k}$. Note that writing for any $\theta \in(0,1)\|\nabla v\|_{L_{2}\left(K^{\prime}\right)}^{2} \leq \theta\|\nabla v\|_{L_{2}\left(K^{\prime}\right)}^{2}+(1-\theta) C 2^{2 j\left(K^{\prime}\right)}\|v\|_{L_{2}\left(K^{\prime}\right)}^{2}$, we could have replaced the constant $\underline{\lambda}_{J^{\prime}}^{-1}$ by $\min _{\theta \in[0,1]} \max \left\{\theta \underline{\lambda}_{J^{\prime}}^{-1}, \frac{C(1-\theta) 2^{2 j\left(J^{\prime}\right)}}{\min _{x \in J^{\prime}}|b(x)|}\right\}$. This would allow us to get better estimates when the diffusion coefficient degenerates and the zero order term is large. We shall dispense though with making these distinctions in what follows. At any rate, employing Young's inequality in combination with 2.6 and a suitable choice of $\sigma>0$ shows that there is indeed a positive $\gamma$ such that

$$
a_{h}(v, v) \geq \frac{1}{2}\left(\sum_{K \in \mathcal{T}_{h}} a(v, v)_{K}+\sum_{e \in \mathcal{E}_{h}}|e|^{-1}\|[v]\|_{L_{2}(e)}^{2}\right)=: \frac{1}{2}\|v\| \|_{h}^{2}, \quad v \in V_{h},
$$

which yields the desired stability.

REMARK 2.1. If we allow $\gamma$ to vary, we should take $\gamma-\sigma \geq 1 / 2$ where for any edge of $K$, the $\sigma$ and hence $\gamma$ should be of the order of $\underline{\lambda}_{K}^{-1}$ up to constants depending on the shape properties of $\mathcal{T}^{0}$ and the degree $k$ of the piecewise polynomials, but not on $h$.

The same type of estimates establishes continuity

$$
\left|a_{h}(v, w)\right| \leq \bar{C} \mid\|v\|\left\|_{h}\right\| w \|_{h}, \quad v, w \in V_{h}
$$

for some uniform constant $\bar{C}$ that could actually be made $\bar{C}=2$ when taking $\gamma$ sufficiently large but again of the order of $\underline{\lambda}_{K}^{-1}$ locally. (In fact, continuity could be established even in a larger infinite dimensional space, see e.g. [10].) Hence, the problem

$$
\text { find } u_{h} \in V_{h} \text { such that } a_{h}\left(u_{h}, v\right)=(f, v), \quad \forall v \in V_{h} \text {, }
$$

has a unique solution, where $a_{h}$ is the symmetric bilinear form defined on $V_{h}$ by (2.4).

Unfortunately, as in the case of conforming discretizations the corresponding linear systems of equations grow increasingly ill-conditioned when the mesh size decreases. For instance, for quasi-uniform $\mathcal{T}_{h}$ the spectral condition numbers of stiffness matrices are known to grow like $h^{-2}$, see e.g. 8 . 
3. Additive Schwarz schemes. In this section we explain why a classical multi-wavelet approach fails to yield optimal preconditioners, and formulate the more general framework for additive Schwarz schemes in the DG context that give rise to uniformly bounded condition numbers for the above type of discretizations under no additional regularity requirements on the solution $u$ of 2.2 .

3.1. A way that does not work. Schwarz type preconditioners for conforming discretizations are closely related to change of basis preconditioners when energy space stable multiscale bases are available [5, 6. To explain this, suppose that $\Psi=\left\{\psi_{i}: i \in \mathcal{I}\right\}$ is a basis of $V_{h}$ and recall that the spectral condition number $\kappa\left(\mathbf{A}_{h}^{\Psi}\right)$ of the stiffness matrix $\mathbf{A}_{h}^{\Psi}:=\left(a_{h}\left(\psi_{i}, \psi_{j}\right)\right)_{i, j \in \mathcal{I}}$ is given by

$$
\kappa\left(\mathbf{A}_{h}^{\Psi}\right)=\left(\sup _{\mathbf{v}} \frac{\mathbf{v}^{T} \mathbf{A}_{h}^{\Psi} \mathbf{v}}{\mathbf{v}^{T} \mathbf{v}}\right)\left(\inf _{\mathbf{v}} \frac{\mathbf{v}^{T} \mathbf{A}_{h}^{\Psi} \mathbf{v}}{\mathbf{v}^{T} \mathbf{v}}\right)^{-1} .
$$

Now according to 2.7, 2.8, writing $\mathbf{v}=\left(v_{i}\right)_{i \in \mathcal{I}}$ we have for $v=\sum_{i \in \mathcal{I}} v_{i} \psi_{i}$,

$$
\frac{\mathbf{v}^{T} \mathbf{A}_{h}^{\Psi} \mathbf{v}}{\mathbf{v}^{T} \mathbf{v}}=\frac{a_{h}(v, v)}{\|\mathbf{v}\|_{\ell^{2}}^{2}} \sim \frac{\|v\|_{h}^{2}}{\|\mathbf{v}\|_{\ell^{2}}^{2}}
$$

This immediately yields the following fact.

REMARK 3.1. If $\Psi$ is stable in the sense that

$$
\left\|\left|v\left\|_{h}^{2} \sim \sum_{i \in \mathcal{I}}\left|v_{i}\right|^{2} \mid\right\| \psi_{i} \|_{h}^{2}\right.\right.
$$

for any $v=\sum_{i \in \mathcal{I}} v_{i} \psi_{i}$ in $V_{h}$, then an optimal preconditioner is obtained by a symmetric diagonal scaling, namely we have

$$
\kappa\left(\mathbf{D A}_{h}^{\Psi} \mathbf{D}\right) \sim 1 \quad \text { with } \quad \mathbf{D}=\operatorname{diag}\left(\left\|\psi_{i}\right\|_{h}^{-1}: i \in \mathcal{I}\right) .
$$

In the conforming case such energy space stable bases are typically derived from $L_{2}$-Riesz multilevel bases by rescaling. In the DG context it is very easy to construct $L_{2}$-stable multilevel bases for $V_{h}$ using the concept of multi-wavelets. One might therefore be tempted to construct a change-of-bases preconditioner by suitably rescaling such a multi-wavelet basis. For such a multilevel basis of $V_{h}$, a refinement $\mathcal{T}_{h^{\prime}}$ of the mesh $\mathcal{T}_{h}$ entails expanding the current basis $\Psi_{h}$ for $V_{h}$ to the larger basis $\Psi_{h^{\prime}}$ by adding a collection of new basis functions (on corresponding locally higher refinement levels) that span a complementary space $W$ of $V_{h}$ so that $V_{h^{\prime}}=V_{h} \oplus W$. In the case of multi-wavelets all basis functions would consist of globally discontinuous piecewise polynomials, and in the present context this turns out to cause a principal problem that we describe now.

Consider a discontinuous basis function $\psi_{i}$ of $\Psi$, and assume that it belongs to some space $V_{h}$. Clearly there is at least one edge $e$ in $\mathcal{E}_{h}$ for which $\left\|\left[\psi_{i}\right]\right\|_{L_{2}(e)}$ is positive. Now, if $\mathcal{T}_{h^{\prime}}$ is a refinement of $\mathcal{T}_{h}$ where $e$ is uniformly refined into $2^{l}$ pieces, we have

$$
\left\|\psi_{i}\right\|_{h^{\prime}}^{2} \geq \sum_{e^{\prime} \in \mathcal{E}_{h^{\prime}}: e^{\prime} \subset e} \frac{1}{\left|e^{\prime}\right|}\left\|\left[\psi_{i}\right]\right\|_{L_{2}\left(e^{\prime}\right)}^{2}=2^{l} \frac{1}{|e|}\left\|\left[\psi_{i}\right]\right\|_{L_{2}(e)}^{2} .
$$

Hence $\left\|\psi_{i}\right\| \|_{h^{\prime}}$ tends to infinity as the edge $e$ is further refined. As a first consequence, this shows that the $\psi_{i}$ will not remain normalized when progressing to higher levels of refinements causing the mesh dependent norm $\||\cdot|\|_{h}$ to change.

This does not exclude yet stability in the sense of 3.1. On the other hand, the energy norm of a continuous $v \in V_{h}$ reduces to $a(v, v)^{1 / 2}$ and hence does not depend on the mesh. If $\psi_{i}$ remains unchanged when expanding the basis of $V_{h}$ to a basis of $V_{h^{\prime}}$, the associated coordinate $v_{i}$ does not change either. The desired stability of $\Psi$ would thus yield

$$
a(v, v)=a_{h}(v, v)=\left\|\left|v\left\|_{h^{\prime}}^{2} \sim \sum_{i^{\prime} \in \mathcal{I}_{h^{\prime}}}\left|v_{i^{\prime}}\right|^{2}\right\| \psi_{i^{\prime}}\left\|_{h^{\prime}}^{2} \geq\left|v_{i}\right|^{2}\right\| \psi_{i} \|_{h^{\prime}}^{2}\right.\right.
$$


which clearly implies that $v_{i}=0$, since otherwise the (constant) quantity $a(v, v)$ would have to tend to infinity with $h^{\prime} \rightarrow 0$.

From this observation we infer that when $\Psi$ is a uniformly stable multilevel basis for the spaces $V_{h}$, then it must have a subset $\Psi^{\mathrm{c}}$ that spans the conforming spaces $V_{h}^{\mathrm{c}}:=V_{h} \cap H_{0}^{1}(\Omega)$. In particular, $\Psi$ and $V_{h}$ respectively decompose into

$$
\Psi=\Psi^{\mathrm{c}} \cup \Psi^{\mathrm{nc}} \quad \text { and } \quad V_{h}=V_{h}^{\mathrm{c}} \oplus V_{h}^{\mathrm{nc}}
$$

in such a way that subsets of $\Psi^{\mathrm{c}}$ and $\Psi^{\mathrm{nc}}$ are stable bases for $V_{h}^{\mathrm{c}}$ and $V_{h}^{\mathrm{nc}}$, respectively, and that the splitting is stable with respect to the discrete energy norm $\||\cdot|\|_{h}$.

This observation that appears to exclude standard multi-wavelets will guide the subsequent developments.

3.2. Additive Schwarz preconditioners. It is of course not necessary to construct stable multilevel bases to produce efficient preconditioners for the problem 2.9p of finding $u_{h} \in V_{h}$ such that

$$
a_{h}\left(u_{h}, v\right)=(f, v), \quad v \in V_{h} .
$$

In fact, the theory of additive Schwarz schemes offers a more flexible framework based on so-called stable splittings for the respective energy space which in turn permit redundant systems, namely frames. Such stable splittings for the DG setting will be constructed in Section 4.3 below. First let us briefly recall the notion of such splittings and under which circumstances they give rise to good Schwarz preconditioners. The collection

$$
\mathcal{S}=\left\{V_{i}\right\}_{i \in \mathcal{I}_{h}}, \quad V_{i} \subset V_{h},
$$

is called a $\left(\||\cdot|\|_{h^{-}}\right)$stable splitting of $V_{h}$, uniformly with respect to all triangulations $\mathcal{T}_{h}$ of the specified type, if $\sum_{i \in \mathcal{I}_{h}} V_{i}=V_{h}$ and

$$
c_{S}\|v\|\left\|_{h}^{2} \leq \inf _{\substack{v_{i} \in V_{i} \\ v=\sum_{i \in \mathcal{I}_{h}} v_{i}}}\left\{\sum_{i \in \mathcal{I}_{h}}\left\|v_{i}\right\|_{h}^{2}\right\} \leq C_{S}\right\| v \|_{h}^{2}, \quad v \in V_{h}
$$

holds with constants $c_{S}, C_{S}$ independent of $\mathcal{T}_{h}$.

With the aid of such stable splittings one can transform equation 3.2 into an equivalent problem which will be uniformly well conditioned.

To this end, the next ingredients are auxiliary inner products on the spaces $V_{i}$, namely

$$
b_{i}(\cdot, \cdot): V_{i} \times V_{i} \rightarrow \mathbb{R}, \quad i \in \mathcal{I}_{h},
$$

yielding norms that are locally equivalent to $\|\mid \cdot\|_{h}$, i.e. satisfy

$$
c_{b}\left\|v_{i}\right\|_{h}^{2} \leq b_{i}\left(v_{i}, v_{i}\right) \leq C_{b}\left\|v_{i}\right\|_{h}^{2}, \quad v_{i} \in V_{i}, i \in \mathcal{I}_{h},
$$

for constants $c_{b}, C_{b}$ depending at most on the maximal degree $\bar{k}$, the shape properties of $\mathcal{T}^{0}$ and possibly on the coefficients in the bilinear form $a(\cdot, \cdot)$ (see 4.37) below for concrete choices).

Finally, define the operators $P_{i}: V_{h} \rightarrow V_{i}$ and elements $f_{i} \in V_{i}$ by

$$
b_{i}\left(P_{i} w, v_{i}\right)=a_{h}\left(w, v_{i}\right), \quad b_{i}\left(f_{i}, v_{i}\right)=\left\langle f, v_{i}\right\rangle, \quad v_{i} \in V_{i}, i \in \mathcal{I}_{h} .
$$

Note that, whenever $V_{i}=\operatorname{span}\left(\theta_{i}\right)$ is a one-dimensional space, the application of $P_{i}$ just amounts to solving a linear equation with a single unknown, namely

$$
P_{i} w=d_{i} \theta_{i} \quad \Longleftrightarrow \quad d_{i}=a_{h}\left(w, \theta_{i}\right) / b_{i}\left(\theta_{i}, \theta_{i}\right) .
$$

The central result we shall use reads then as follows, see e.g. [14, 7]. 
Theorem 3.1. Define $P_{h}: V_{h} \rightarrow V_{h}$ and $\bar{f}_{h} \in V_{h}$ by

$$
P_{h}:=\sum_{i \in \mathcal{I}_{h}} P_{i}, \quad \bar{f}_{h}:=\sum_{i \in \mathcal{I}_{h}} f_{i}
$$

Then the problem (3.2) is equivalent to the operator equation

$$
P_{h} u=\bar{f}_{h} .
$$

Moreover, if (3.3) and (3.4) hold, then the spectral condition number of the symmetric positive definite (with respect to the inner product $\left.a_{h}(\cdot, \cdot)\right)$ operator $P_{h}$ can be bounded by

$$
\kappa\left(P_{h}\right) \leq 2 \bar{C} \frac{C_{b} C_{S}}{c_{b} c_{S}}
$$

thus by a constant independent of $\mathcal{T}_{h}$, see 2.8, 3.3 and 3.4. Recall that $\kappa\left(P_{h}\right)=\frac{\lambda_{\max }\left(P_{h}\right)}{\lambda_{\min }\left(P_{h}\right)}$, where

$$
\lambda_{\max }\left(P_{h}\right)=\sup _{\substack{v \in V_{h} \\ v \neq 0}} \frac{a_{h}\left(P_{h} v, v\right)}{a_{h}(v, v)} \quad \text { and } \quad \lambda_{\min }\left(P_{h}\right)=\inf _{\substack{v \in V_{h} \\ v \neq 0}} \frac{a_{h}\left(P_{h} v, v\right)}{a_{h}(v, v)} .
$$

Obviously the complexity of applying the operator $P_{h}$ in some iterative procedure has linear complexity when the dimension of the subspaces $V_{i}$ is uniformly bounded. Such stable splittings will be constructed in Sections 4.3 and 5 below, see in particular Corollary 5.3 for one specific realization.

Now, to interprete the above result let us view 3.2 as the operator equation

$$
L_{h} u_{h}=f, \quad \text { where } L_{h} \text { is defined by }\left\langle L_{h} w, v\right\rangle=a_{h}(w, v), \quad w, v \in V_{h} .
$$

Moreover, defining $L_{i}: V_{i} \rightarrow V_{i}$ by $b_{i}\left(w_{i}, v_{i}\right)=\left\langle L_{i} w_{i}, v_{i}\right\rangle$ (i.e. $L_{i}^{-1}$ is the approximate inverse of the restriction of $L_{h}$ to $\left.V_{i}\right)$, and denoting by $Q_{i}: V_{h} \rightarrow V_{i}$ the $V_{i}$-orthogonal projection, we have by definition,

$$
P_{i}=L_{i}^{-1} Q_{i} L_{h}
$$

Therefore, we can write as usual

$$
P_{h} u=\left(\sum_{i \in \mathcal{I}_{h}} L_{i}^{-1} Q_{i}\right) L_{h} u \quad \text { and } \quad \bar{f}=\left(\sum_{i \in \mathcal{I}_{h}} L_{i}^{-1} Q_{i}\right) f
$$

i.e. the operator

$$
\mathcal{C}:=\sum_{i \in \mathcal{I}_{h}} L_{i}^{-1} Q_{i}
$$

can be viewed as a preconditioner.

4. Stable splittings for the DG method. This section is devoted to the construction and analysis of stable multilevel splittings for the DG method that can be used in Theorem 3.1. There are two ingredients that are perhaps worth emphasizing.

- The first one is that stable splittings for $V_{h}$ can simply be assembled from stable splittings for an appropriate conforming and nonconforming part of $V_{h}$, respectively. The appropriateness will hinge on certain admissible averaging operators that are required to satisfy a Jackson estimate.

- Second, the locality of such an estimate, however, turns out to impose a slightly stronger notion of gradedness (see Definition 4.1 below) for the underlying meshes (without actually inflating the complexity, see Remark 4.2 below) which we shall therefore address first. 
4.1. Admissible triangulations - extended grading. We have already defined a standard grading requirement prohibiting an edge to have more than one hanging vertex. The grading that we shall have to impose on the triangulations will actually be slightly stronger, but essentially keeps the complexity equivalent, see Remark 4.2 below.

To limit technicalities we shall only consider triangulations that are obtained by local subdivisions into four congruent subtriangles, starting from a conforming and shape regular coarse $\mathcal{T}^{0}$, as in Figure 2.1. As a consequence, every element of $\mathcal{T}_{h}$ has a refinement level $j(K), j(K)=0$ meaning that $K \in \mathcal{T}^{0}$. From the shape regularity of $\mathcal{T}_{h}$ (inherited from that of $\mathcal{T}^{0}$ ), one then easily infers

$$
|K|:=\operatorname{meas}(K) \sim\left(\left.h\right|_{K}\right)^{2} \sim 2^{-2 j(K)} \quad \text { for any } K \in \mathcal{T}_{h}
$$

with constants that depend only on the coarse $\mathcal{T}^{0}$.

DEFINITION 4.1 (graded triangulations). A triangulation $\mathcal{T}_{h}$ obtained from refining a conforming coarse $\mathcal{T}^{0}$ as described above is called graded, if every hanging vertex $n$ is the midpoint of an edge spanned by two vertices, denoted by $n^{\prime}$ and $n^{\prime \prime}$ in Figure 4.1. left, and if in such a case, $n^{\prime}$ and $n^{\prime \prime}$ are never hanging vertices themselves.

Note that this grading requirement implies the usual one, i.e. there is at most one hanging vertex per edge.
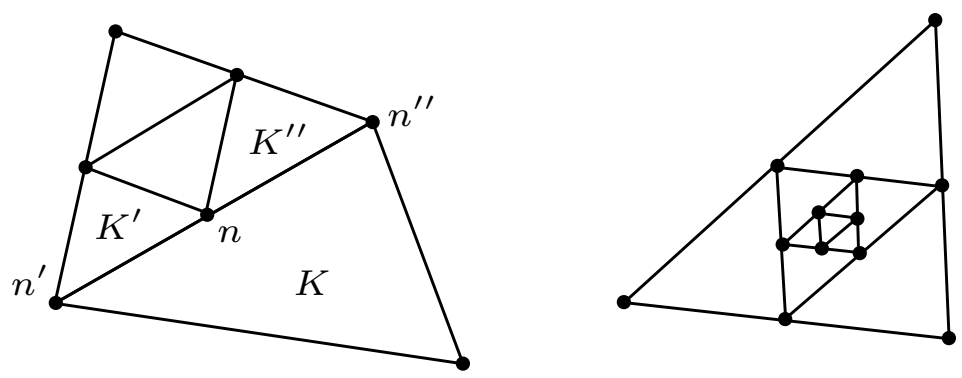

FIG. 4.1. For $\mathcal{T}_{h}$ be graded (the right one is not), $n^{\prime}$ and $n^{\prime \prime}$ must be regular vertices.

REMARK 4.1 (necessity of the (extended) grading). The above grading requirement is necessary for Theorem 1.1 (and further similar splittings) to hold.

To see the validity of the above claim consider one triangulation $\mathcal{T}_{h}$ that has been obtained from one single coarse triangle $\mathcal{T}^{0}=\left\{K_{0}\right\}$ by recursively refining the central child at each level up to some $j_{h}$, as shown in Figure 4.1, right. Clearly, such a $\mathcal{T}_{h}$ satisfies the "classical" grading requirement permitting only one hanging vertex per edge, but not the above stronger one. In particular, all its vertices except those on $\partial \Omega$ are hanging, so that $H_{0}^{1}(\Omega) \cap \mathbb{P}_{1}\left(\mathcal{T}_{h}\right)$ only contains 0 . Hence Theorem 1.1 would imply here that for any basis $\left\{\phi_{i}: i \in \mathcal{I}_{h}\right\}$ of $V_{h}:=\mathbb{P}_{k}\left(\mathcal{T}_{h}\right)$ consisting of local polynomial pieces normalized in $L_{\infty}$, one has

$$
\|\| v \|_{h}^{2} \sim \sum_{i \in \mathcal{I}_{h}}\left|d_{i}\right|^{2} \quad \text { for every } \quad v=\sum_{i \in \mathcal{I}_{h}} d_{i} \phi_{i} \in V_{h},
$$

with constants independent of the refinement level $j_{h}$. Consider now the piecewise constant function $v=\chi_{K}$, where $K$ is the central triangle on level two. If the $\phi_{i}$ are interpolatory, like in a local Lagrange basis, one has $d_{i}=1$ for all but finitely many indices $i$ and the right hand side in 4.2 grows proportionally to $j_{h}$. On the other hand, since $v$ is continuous inside $K$, it can be seen from the definition of the energy norm, given in Section 2.1, see (2.7), that $\|v\| \|_{h}$ doesn't depend on the further refinements of $\mathcal{T}_{h}$, which clearly contradicts 4.2 .

We shall next give an algorithmic characterization of the grading property in Definition 4.1 Since the triangulations $\mathcal{T}_{h}$ of interest result from (possibly local) refinements, such a refinement history defines a tree $\widehat{\mathcal{T}}_{h}$, where $\mathcal{T}_{h}$ is the set of leaves (i.e. final nodes) of $\widehat{\mathcal{T}}_{h}$. We denote by $\boldsymbol{P}(K)$ and $\boldsymbol{C}(K)$ the parent and set of children, respectively, of a given triangle $K$. This means any child $K^{\prime} \in C(K)$ is obtained by subdividing its parent $K=\boldsymbol{P}\left(K^{\prime}\right)$ once, according to the given rule. By $\boldsymbol{P}^{2}(K)=\boldsymbol{P}(\boldsymbol{P}(K))$ we denote the grandfather of $K$ when $j(K) \geq 2$. 
Also, by $\mathcal{T}^{j}$ we denote the $j$ th uniform dyadic refinement of $\mathcal{T}^{0}$. Clearly, for any level $j$ this is a partition of $\Omega$. Given any triangle $K$ in $\mathcal{T}_{h}$, we then let its neighbors $\boldsymbol{N}(K)$ be defined as the collection of triangles at level $j(K)$, i.e. belonging to $\mathcal{T}^{j(K)}$ (but not necessarily to $\widehat{\mathcal{T}}_{h}$ ) that share an edge with $K$. Finally, $\boldsymbol{N}^{2}(K):=\cup_{K^{\prime} \in \boldsymbol{N}(K)} \boldsymbol{N}\left(K^{\prime}\right)$ consists of the neighbors of the neighbors of $K$. In these terms a constructive formulation of the grading property reads as follows.

REMARK 4.2 (algorithmic formulation of the grading requirement). A triangle $K$ in $\mathcal{T}_{h}$ is said to have the $\mathbf{G}$-property if it is of level $j(K) \leq 1$ or if

- in the case that $\boldsymbol{P}(K)$ is not the central child of $\boldsymbol{P}^{2}(K)$, every triangle of $\boldsymbol{N}(\boldsymbol{P}(K))$ is in the tree $\widehat{\mathcal{T}}_{h}$, or

- in the case that $\boldsymbol{P}(K)$ is the central child of $\boldsymbol{P}^{2}(K)$, every triangle of $\boldsymbol{N}^{2}(\boldsymbol{P}(K))$ is in the tree $\widehat{\mathcal{T}}_{h}$.

Then the following properties hold, whose proofs are left to the reader.

1. A triangulation $\mathcal{T}_{h}$ is graded in the sense of Definition 4.1 if and only if all its elements satisfy the $\mathbf{G}$-property.

2. Given any multilevel triangulation $\mathcal{T}_{h}$, its smallest graded refinement is built by imposing recursively (starting from the highest levels) the $\mathbf{G}$-property on all its elements.

Therefore the $\mathbf{G}$-property is a practical tool for constructing graded triangulations. Moreover, it can be checked that the set

$$
\widehat{\mathcal{T}}_{h}^{*}:=\widehat{\mathcal{T}}_{h} \cup \bigcup_{K \in \widehat{\mathcal{T}}_{h}} \boldsymbol{C}\left(\boldsymbol{N}^{2}(\boldsymbol{P}(K))\right)
$$

is indeed a tree, and that the corresponding set of leaves $\mathcal{T}_{h}^{*}$ is a refinement of $\mathcal{T}_{h}$ in which any triangle has the $\mathbf{G}$-property. $\mathcal{T}_{h}^{*}$ is then a graded refinement of $\mathcal{T}_{h}$, and it is a standard exercise (see for instance Lemma 2.4 in [4]) to derive from (4.3) the following complexity estimates

$$
\#\left(\mathcal{T}_{h}^{*}\right) \sim \#\left(\widehat{\mathcal{T}}_{h}^{*}\right) \sim \#\left(\widehat{\mathcal{T}}_{h}\right) \sim \#\left(\mathcal{T}_{h}\right)
$$

which holds with uniform constants. Thus, imposing our (extended) grading keeps the complexity of adaptive triangulations up to uniform constants.

We shall henceforth assume that $\mathcal{T}_{h}$ is graded in the sense of Definition 4.1

4.2. Admissible conforming subspaces. Given a graded triangulation $\mathcal{T}_{h}$, it remains to construct energy stable splittings to be used in Theorem 3.1. Motivated by the observations made in Section 3.1 . we shall first look decomposing $V_{h}$ into a conforming and nonconforming part $V_{h}^{\text {c }}$ and $V_{h}^{\text {nc }}$, respectively. However, it turns out that in the more general framework offered by Schwarz preconditioners, even the meaning of $V_{h}^{\mathrm{c}}$ can be relaxed in that it need not exhaust all of $V_{h} \cap H_{0}^{1}(\Omega)$. Different conforming subspaces will be seen to lead to different versions of preconditioners that are all asymptotically optimal in the sense of uniformly bounded condition numbers.

What matters is that the conforming part is the range of a suitable averaging projector $\mathcal{A}$ into some subspace of $V_{h} \cap H_{0}^{1}(\Omega)$. In this section we formulate the key property that qualifies the averaging projector as suitable, namely a certain local Jackson-type estimate involving for each $K \in \mathcal{T}_{h}$ the localized energy norms

$$
\|v\|_{h, \omega}^{2}:=\sum_{K \in \mathcal{T}_{h}: K \subset \omega} a(v, v)_{K}+\sum_{e \in \mathcal{E}_{h}: e \subset \omega}|e|^{-1}\|[v]\|_{L_{2}(e)}^{2}
$$

for certain neighborhoods $\omega=\omega(K) \subset \Omega$ of any $K \in \mathcal{T}_{h}$.

To define $\omega(K)$ we need some further preparations and notational conventions. The set of vertices viz. first degree nodes will be denoted by

$$
\mathcal{N}_{1}(K):=\{\text { vertices of } K\} \quad \text { and } \quad \mathcal{N}_{h, 1}:=\bigcup_{K \in \mathcal{T}_{h}} \mathcal{N}_{1}(K) .
$$

In connection with higher order elements we shall have to consider later corresponding sets of higher $k$ th degree nodes that will be denoted by $\mathcal{N}_{k}(K)$, see Section 4.3 
Moreover, it will be convenient to set for any closed domain $D$

$$
\mathcal{T}_{h}(D):=\left\{K \in \mathcal{T}_{h}: K \cap D \neq \emptyset\right\} .
$$

Similarly we define the sets $\mathcal{N}_{h, 1}(D)$ and $\mathcal{E}_{h}(D)$ for mesh elements touching $D$. For instance, $\mathcal{T}_{h}(n)$ consists of the triangles that share the vertex $n$ (including hanging vertices). Note that when an edge of a triangle $K$ contains a hanging vertex the collections $\mathcal{N}_{1}(K)$ and $\mathcal{N}_{h, 1}(K)$ differ by that hanging vertex. Unfortunately, due to hanging vertices, straightforward neighborhoods based on these notions do not suffice and the construction of the domains $\omega(K)$ appearing in the announced Jackson estimate will actually rely on some extended sets of mesh quantities.

To this end, recall that for any vertex $n$ there are two possibilities: either it is hanging, i.e. there is one triangle $K$ in $\mathcal{T}_{h}(n)$ for which $n$ is not a vertex, and then $n$ is the midpoint of one edge $e=\left[n^{\prime}, n^{\prime \prime}\right]$ of $K$, see Figure 4.1, or it is not. With these notations we first define

$$
\mathcal{N}_{h, 1}^{*}(n):= \begin{cases}\left\{n, n^{\prime}, n^{\prime \prime}\right\} & \text { if } n \text { is hanging, } \\ \{n\} & \text { otherwise, i.e. if } n \text { is regular. }\end{cases}
$$

Recall that the grading we impose on $\mathcal{T}_{h}$ precisely means that both $n^{\prime}$ and $n^{\prime \prime}$ are regular, see Definition 4.1. For any triangle $K \in \mathcal{T}_{h}$ we then set

$$
\begin{aligned}
\mathcal{N}_{h, 1}^{*}(K) & :=\bigcup_{n \in \mathcal{N}_{1}(K)} \mathcal{N}_{h, 1}^{*}(n) \quad \subset \mathcal{N}_{h, 1}, \\
\mathcal{E}_{h}^{*}(K) & :=\bigcup_{n \in \mathcal{N}_{h, 1}^{*}(K)} \mathcal{E}_{h}(n) \quad \subset \mathcal{E}_{h}
\end{aligned}
$$

and

$$
\mathcal{T}_{h}^{*}(K):=\bigcup_{n \in \mathcal{N}_{h, 1}^{*}(K)} \mathcal{T}_{h}(n) \subset \mathcal{T}_{h}
$$

and finally define the domain

$$
\omega(K):=\bigcup_{K^{\prime} \in \mathcal{T}_{h}^{*}(K)} K^{\prime} \subset \Omega
$$

as the union of triangles that are in contact with the extended set of vertices $\mathcal{N}_{h, 1}^{*}(K)$. An illustration is presented in Figure 4.2, where the sets $\mathcal{N}_{h, 1}^{*}(K), \mathcal{E}_{h}^{*}(K)$ and $\mathcal{T}_{h}^{*}(K)$ are represented by white vertices, bold edges and gray triangles, respectively.

Note in particular that the above definitions yield

$$
\sum_{K^{\prime} \in \mathcal{T}_{h}^{*}(K)} a(v, v)_{K^{\prime}}+\sum_{e \in \mathcal{E}_{h}^{*}(K)}|e|^{-1}\|[v]\|_{L_{2}(e)}^{2} \leq\|v\|_{h, \omega(K)}^{2} .
$$

We are now prepared to formulate the key property of averaging projectors $\mathcal{A}$ that will be crucial for the subsequent developments.

Property 4.3. A linear projector $\mathcal{A}$ that takes $V_{h}$ into $V_{h} \cap H_{0}^{1}(\Omega)$ is called admissible if for any $v \in V_{h}$ one has the following Jackson estimate

$$
\|(I-\mathcal{A}) v\|_{L_{2}(K)} \leq C^{*} 2^{-j(K)}\|v\| \|_{h, \omega(K)}, \quad K \in \mathcal{T}_{h},
$$

with a constant $C^{*}$ that may depend on the shape properties of $\mathcal{T}^{0}$ and on $\bar{k}$ (and on concrete realizations of $\gamma$ ). Note that the domains $\omega(K)$ defined by (4.8) are local (closed) neighborhoods of the triangles $K \in \mathcal{T}_{h}$ satisfying a bounded overlapping property, i.e.

$$
\sup _{K \in \mathcal{T}_{h}} \#\left(\left\{K^{\prime} \in \mathcal{T}_{h}: \omega(K) \cap \omega\left(K^{\prime}\right) \neq \emptyset\right\}\right) \lesssim 1
$$




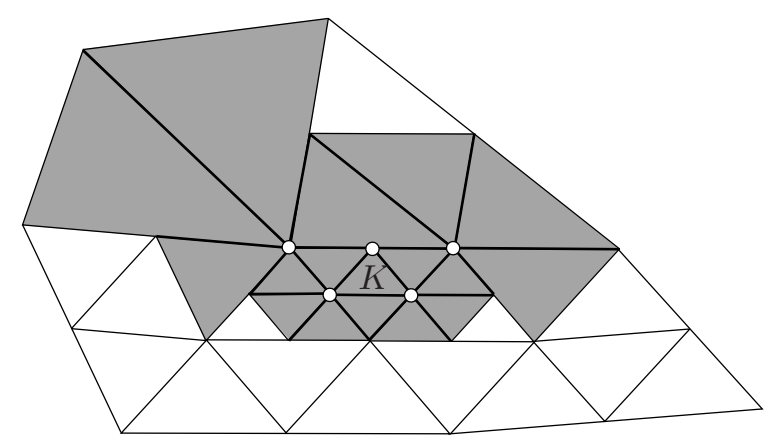

FIG. 4.2. The neighborhood $\omega(K)$ consists of the union of the (gray) triangles in $\mathcal{T}_{h}^{*}(K)$.

holds with a constant that depends only on $\mathcal{T}^{0}$.

We postpone the concrete construction of admissible $\mathcal{A}$ and the verification of Property 4.3 to Section 4 and discuss first the consequences regarding stable splittings.

The significance of Property 4.3 lies in the following two consequences, summarized in Propositions 4.2 and 4.3 . The first one concerns the energy stability of an admissible $\mathcal{A}$.

Proposition 4.2. Assume that $\mathcal{A}$ satisfies the Jackson estimate (4.10). Then one has

$$
\|(I-\mathcal{A}) v\|_{h} \lesssim\|\| v\left\|_{h}, \quad\right\| \mid \mathcal{A} v\left\|_{h} \lesssim\right\| v \|_{h}, \quad v \in V_{h},
$$

where the constants depend on $\bar{k}$, the shape properties of $\mathcal{T}^{0}$ as well as on $C^{*}$ and $\bar{C}_{a}$ defined in (4.14) below.

As a consequence we have

$$
\|v\|\left\|_{h} \leq\right\| \mid \mathcal{A} v\|\|_{h}+\|(I-\mathcal{A}) v\|_{h} \leq C_{\mathcal{A}}\|v\|_{h},
$$

with the above dependence of the constant $C_{\mathcal{A}}$ in the upper bound.

Proof: Since the jumps of $(I-\mathcal{A}) v$ equal the jumps of $v$ we need only estimate $\sum_{K \in \mathcal{T}_{h}} a((I-\mathcal{A}) v,(I-$ A) $v)_{K}$. To keep track of the dependence of the various constants, recall that $\bar{\lambda}_{K}$ is defined by 2.5 and let

$$
C_{a, K}:=2 \max \left\{\bar{\lambda}_{K}, 2^{-2 j(K)}\|b\|_{L_{\infty}(K)}\right\} \text { and } \bar{C}_{a}:=\max _{K \in \mathcal{T}_{h}} C_{a, K} \lesssim C_{a},
$$

see 2.3 . Since $(I-\mathcal{A}) v$ is a polynomial on $K$, a standard inverse estimate yields

$$
a((I-\mathcal{A}) v,(I-\mathcal{A}) v)_{K} \leq c C_{a, K} 2^{2 j(K)}\|(I-\mathcal{A}) v\|_{L_{2}(K)}^{2} \lesssim C^{*} C_{a, K}\|v\|_{h, \omega(K)}^{2},
$$

where $c$ depends on $k$ and $\mathcal{T}^{0}$ and where we have used 4.10 again in the last step. On account of the bounded overlap of the $\omega(K), K \in \mathcal{T}_{h}$ (see (4.11)), and since $\|\mathcal{A} v\|_{h} \leq\|v\|\left\|_{h}+\right\|(I-\mathcal{A}) v \|_{h}$, this concludes the proof.

Thus, defining

$$
V_{h}^{\mathrm{c}}:=\mathcal{A} V_{h} \quad \text { and } \quad V_{h}^{\mathrm{nc}}:=(I-\mathcal{A}) V_{h},
$$

as possible candidates for conforming and nonconforming parts of $V_{h}$, the above observation 4.13 already indicates that a stable splitting for all of $V_{h}$ of the form (3.3) can be composed of stable splittings for the individual subspaces $V_{h}^{\mathrm{c}}$ and $V_{h}^{\text {nc }}$, respectively. In fact, this would allows us to invoke known splittings for conforming spaces, see e.g. [3, 5, 7, 14, and Section 5.1.1.

Now, the second consequence of Property 4.3 is a localization of $V_{h}^{\text {nc }}$ that will simplify the identification of stable splittings for the nonconforming part. In what follows we shall make frequent use of the 
following simple consequences of standard trace inequalities, rescaling arguments and the fact that all norms on a fixed finite dimensional space are equivalent.

REMARK 4.4. For any polynomial $P \in \mathbb{P}_{k}$, any $K \in \mathcal{T}_{h}$ and any edge $e$ of $K$, the following relations hold.

(i) One has

$$
\|P\|_{L_{\infty}(e)} \sim 2^{j(K) / 2}\|P\|_{L_{2}(e)},
$$

where the constants depend only on the degree $k$ of $P$ and on $\mathcal{T}_{0}$.

(ii) There exists a constant $c$ depending only on the degree $k$ of $P$ and on $\mathcal{T}^{0}$ such that

$$
\left\|\chi_{K} P\right\|_{h} \leq c\left(C_{a, K}+1\right)^{\frac{1}{2}}\left\{\begin{array}{l}
2^{j(K)}\|P\|_{L_{2}(K)}, \\
\|P\|_{L_{\infty}(K)}
\end{array}\right.
$$

where the constant $c$ depends only $k$ and the shape properties of $\mathcal{T}^{0}$ and $C_{a, K}$ is given by (4.14). Observe then that splittings for $V_{h}^{\text {nc }}$ will indeed take a simple form due to localization.

Proposition 4.3. Assume that $\mathcal{A}$ is admissible, i.e. satisfies (4.10). Then there exists a constant $C_{0}$, depending only on $\bar{k}$, the shape properties of $\mathcal{T}^{0}$, on $\bar{C}_{a}$ defined in (4.14), and on the constant $C^{*}$ in (4.10) such that for $w^{K}:=\chi_{K} w$

$$
\|w \mid\|_{h}^{2} \leq \sum_{K \in \mathcal{T}_{h}}\left\|w^{K}\right\|_{h}^{2} \leq C_{0}\|w\|_{h}^{2}, \quad w \in V_{h}^{\mathrm{nc}}:=(I-\mathcal{A}) V_{h} .
$$

Proof: The lower inequality in 4.18 is due to the fact that for any edge $e \subset K \cap K^{\prime}$ one has

$$
\|[w]\|_{L_{2}(e)}^{2} \leq\left\|w^{K}\right\|_{L_{2}(e)}^{2}+\left\|w^{K^{\prime}}\right\|_{L_{2}(e)}^{2} .
$$

As for the upper inequality, since $w \in V_{h}^{\mathrm{nc}}$ and $w^{K}$ is a polynomial on $K$, we infer from 4.17 and 4.10 (noticing $(I-\mathcal{A}) w=w)$ that

$$
\left\|w^{K}\right\|_{h}^{2} \lesssim\left(C_{a, K}+1\right) 2^{2 j(K)}\left\|w^{K}\right\|_{L_{2}(K)}^{2} \lesssim\left(C_{a, K}+1\right) C^{*}\|w\|_{h(\omega(K))}^{2},
$$

where $C_{a, K}$ can be bounded by $C_{a}$ from 2.3 . The upper inequality follows now again from (4.11).

4.3. Energy stable splittings for $V_{h}$. As mentioned above, the stability of $\mathcal{A}$ asserted by Proposition 4.2 implies that stable splittings for the individual parts $V_{h}^{\mathrm{c}}$ and $V_{h}^{\text {nc }}$ give rise to stable splittings for all of $V_{h}$. Moreover, the localization in $V_{h}^{\mathrm{nc}}$ given by Proposition 4.3 together with the fact that the frame for the nonconforming part need actually not be contained in $V_{h}^{\mathrm{nc}}$ greatly simplifies finding such a complementary frame.

THEOREM 4.4. Let $\left\{V_{i}^{\mathrm{c}}\right\}_{i \in \mathcal{I}_{h}^{\mathrm{c}}}$ be a stable splitting for $V_{h}^{\mathrm{c}}$, i.e.

$$
c_{1} a(v, v) \leq \inf _{\substack{v_{i} \in V_{i}^{\mathrm{c}} \\ v=\sum_{i \in \mathcal{I}_{h}^{\mathrm{c}}}}}\left\{\sum_{i \in \mathcal{I}_{h}^{\mathrm{c}}} a\left(v_{i}, v_{i}\right)\right\} \leq C_{1} a(v, v), \quad v \in V_{h}^{\mathrm{c}},
$$

holds for some constants $c_{1}, C_{1}$ independent of $\mathcal{T}_{h}$. Then one has for any $v \in V_{h}$

$$
c_{S}\|v\|_{h}^{2} \leq \inf _{\substack{v_{K}, v_{i} \\ v=\sum_{K \in \mathcal{T}_{h}} v_{K}+\sum_{i \in \mathcal{I}_{h}^{c}} v_{i}}}\left\{\sum_{K \in \mathcal{T}_{h}}\left\|v_{K}\right\|_{h}^{2}+\sum_{i \in \mathcal{I}_{h}^{c}} a\left(v_{i}, v_{i}\right)\right\} \leq C_{S}\|v\| \|_{h}^{2},
$$

where the $v_{K}$ and $v_{i}$ belong to $\chi_{K} \mathbb{P}_{k}(K)$ and $V_{i}^{\mathrm{c}}$, respectively, and $c_{S}=\left(\max \left\{2, c_{1}^{-1}\right\}\right)^{-1}, C_{S}=C_{\mathcal{A}} \max \left\{C_{0}, C_{1}\right\}$.

Thus the collection

$$
\mathcal{S}=\left\{\chi_{K} \mathbb{P}_{k}(K): K \in \mathcal{T}_{h}\right\} \cup\left\{V_{i}^{\mathrm{c}}: i \in \mathcal{I}_{h}^{\mathrm{c}}\right\}
$$


is a stable splitting for $V_{h}$ in the sense of 3.3.).

Proof: By definition of the infimum we have

$$
\begin{aligned}
\inf _{\substack{v_{K}, v_{i} \\
v=\sum_{K \in \mathcal{T}_{h}} v_{K}+\sum_{i \in \mathcal{I}_{h}^{c}} v_{i}}}\left\{\sum_{K \in \mathcal{T}_{h}}\left\|v_{K}\right\|_{h}^{2}+\sum_{i \in \mathcal{I}_{h}^{c}} a\left(v_{i}, v_{i}\right)\right\} \\
\leq \inf _{\substack{v_{K} \\
(I-\mathcal{A}) v=\sum_{K \in \mathcal{T}_{h}} v_{K}}}\left\{\sum_{K \in \mathcal{T}_{h}}\left\|v_{K}\right\|_{h}^{2}\right\}+\inf _{\substack{v_{i} \\
\mathcal{A} v=\sum_{i \in \mathcal{I}_{h}^{c}} v_{i}}}\left\{\sum_{i \in \mathcal{I}_{h}^{c}} a\left(v_{i}, v_{i}\right)\right\} \\
\leq C_{0}\|(I-\mathcal{A}) v\|_{h}^{2}+C_{1}\|\mathcal{A} v\|_{h}^{2} \leq C_{S}\|v\|_{h}^{2},
\end{aligned}
$$

where we have used Proposition 4.3 and 4.20 in the second step (remember that $a(\cdot, \cdot)$ and $\|\cdot\|_{h}^{2}$ coincide on $V_{h}^{\mathrm{c}}$ ) and Proposition 4.2 in the last step. Thus $C_{S}=C_{\mathcal{A}} \max \left\{C_{0}, C_{1}\right\}$. This confirms the upper bound in 4.21.

In order to confirm the other direction consider any expansion

$$
v=\sum_{K \in \mathcal{T}_{h}} \tilde{v}_{K}+\sum_{i \in \mathcal{I}_{h}^{c}} \tilde{v}_{i}=: v_{1}+v_{2}
$$

with $\tilde{v}_{K} \in \chi_{K} \mathbb{P}_{k}(K)$ and $\tilde{v}_{i} \in V_{i}^{\mathrm{c}}$. Writing first

$$
\|v\|_{h}^{2} \leq 2\left(\left\|v_{1}\right\|_{h}^{2}+\left\|v_{2}\right\|_{h}^{2}\right)
$$

we note that by 4.20

$$
\left\|v_{2}\right\|_{h}^{2}=a\left(v_{2}, v_{2}\right) \leq c_{1}^{-1} \inf _{\substack{v_{i} \\ v_{2}=\sum_{i \in \mathcal{I}_{h}^{\mathrm{c}}}}}\left\{\sum_{i \in \mathcal{I}_{h}^{\mathrm{c}}} a\left(v_{i}, v_{i}\right)\right\} \leq c_{1}^{-1} \sum_{i \in \mathcal{I}_{h}^{\mathrm{c}}} a\left(\tilde{v}_{i}, \tilde{v}_{i}\right),
$$

while as in the proof of Proposition 4.3 one has for any $v_{1}=\sum_{K \in \mathcal{T}-h} \tilde{v}_{K} \in V_{h}$

$$
\left\|v_{1}\right\|_{h}^{2} \leq \sum_{K \in \mathcal{T}_{h}}\left\|\tilde{v}_{K}\right\|_{h}^{2}
$$

Thus, we can combine 4.25 and 4.26 to conclude that

$$
\|v\|_{h}^{2} \leq 2\left(\sum_{K \in \mathcal{T}_{h}}\left\|\tilde{v}_{K}\right\|_{h}^{2}+c_{1}^{-1} \sum_{i \in \mathcal{I}_{h}^{c}} a\left(\tilde{v}_{i}, \tilde{v}_{i}\right)\right) .
$$

Since the decomposition of $v$ was arbitrary we have shown the lower inequality in 4.21 for $c_{S}=$ $\frac{1}{2}\left(\max \left\{1, c_{1}^{-1}\right\}\right)^{-1}$.

Theorem 4.4 covers various possible specifications of splittings which we shall begin to discuss now. Recall first that, in principle, energy stable splittings for conforming finite element spaces are well understood. Of course, a concrete identification of collections $\left\{V_{i}^{c}\right\}_{i \in \mathcal{I}_{h}^{c}}$ depends on the concrete realization of an admissible projector $\mathcal{A}$, i.e. on the specific subspace $\mathcal{A} V_{h}$. We postpone this issue to Section 5 .

At this point we note first that the subspaces $V_{K}^{\text {nc }}:=\chi_{K} \mathbb{P}_{k}(K)$ are of uniformly bounded finite dimension depending only on $\bar{k}$ and therefore could be further broken down. In fact, any (reasonable) basis for $V_{h}$ will be seen to be an energy stable frame for $V_{h}^{\text {nc }}$. To describe such a basis it is convenient to introduce the canonical $k$-mesh

$$
\mathcal{N}_{k}(K):=\left\{p=p_{\beta}:=\frac{1}{k} \sum_{j=0}^{2} \beta_{j} n_{j}: \beta \in \mathbb{Z}_{+}^{3}, \sum_{j=0}^{2} \beta_{j}=k\right\},
$$




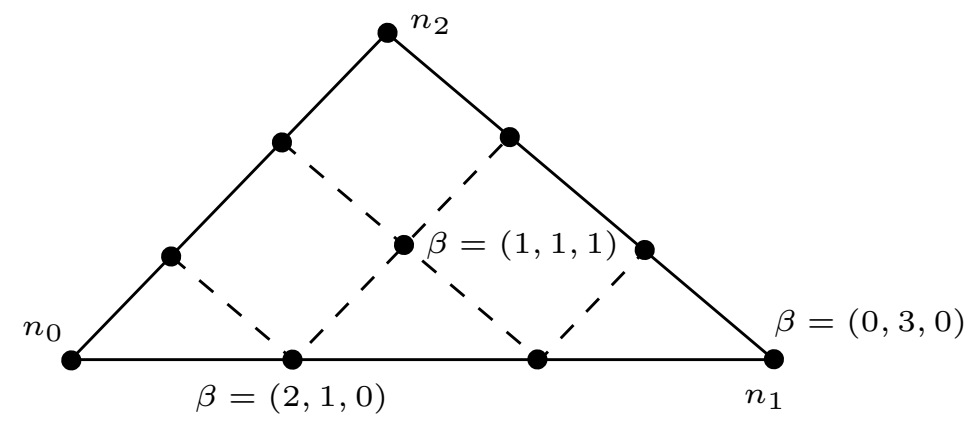

FIG. 4.3. Canonical mesh for $k=3, \mathcal{N}_{3}(K)$.

induced on the triangle $K$, see Figure 4.3 . Of course, for $k=1$ we simply have

$$
\mathcal{N}_{1}(K)=\left\{n_{0}, \ldots, n_{2}\right\},
$$

the set of vertices of $K$ itself, as used before. Note that the $\beta / k$ are the barycentric coordinates of the mesh points $p=p_{\beta}$, a fact that will be used later again. Clearly we have $\operatorname{dim}\left(\mathbb{P}_{k}\right)=\# \mathcal{N}_{k}(K)$.

Now let $\left\{P_{p}: p \in \mathcal{N}_{k}(\hat{K})\right\}$ be a fixed basis for $\mathbb{P}_{k}$, where $\hat{K}$ is the standard triangle with vertices $(0,0),(1,0),(0,1)$, and define for $K=F \hat{K} \in \mathcal{T}_{h}, F=F_{K}$ affine,

$$
\phi_{K, p}:=\chi_{K} P_{p} \circ F_{K}^{-1}, \quad p \in \mathcal{N}_{k}(K) .
$$

Obviously, $\Phi_{h}:=\left\{\phi_{K, p}: p \in \mathcal{N}_{k}(K), K \in \mathcal{T}_{h}\right\}$ is a basis for $V_{h}$. Later, the reference polynomials $P_{p}$ will be (essentially) normalized in $L_{\infty}$ (or in $H^{1}$, which is equivalent in $\mathbb{R}^{2}$ ). Thus one has

$$
\left\|P_{p}\right\|_{L_{\infty}(\hat{K})} \sim 1, \quad\left\|\left\{a_{p}\right\}_{p \in \mathcal{N}_{k}(\hat{K})}\right\|_{\ell_{\infty}} \sim\left\|\sum_{p \in \mathcal{N}_{k}(\hat{K})} a_{p} P_{p}\right\|_{L_{\infty}(\hat{K})},
$$

with constants depending only on $k$ (and the specific choice of the polynomial basis).

Theorem 4.4 yields then the following general result, where it is seen that a complementary frame is simply given by a union of local polynomial basis functions that do not need to belong to the nonconforming part $V_{h}^{\text {nc }}$ but only to span it.

COROllary 4.5. Assume that $\mathcal{A}$ is admissible (i.e. satisfies the Jackson estimate [4.10)) and let $\left\{V_{i}^{\mathrm{c}}: i \in \mathcal{I}_{h}^{\mathrm{c}}\right\}$ be any energy stable splitting for $V_{h}^{\mathrm{c}}:=\mathcal{A} V_{h}$. Moreover, let the basis functions $\phi_{K, p}$ be constructed as above, i.e. they satisfy (4.28), and assume that the set of indices

$$
\mathcal{I}_{h}^{\mathrm{nc}} \subseteq\left\{i=(K, p): p \in \mathcal{N}_{k}(K), K \in \mathcal{T}_{h}\right\}
$$

satisfies

$$
V_{h}^{\mathrm{nc}}:=(I-\mathcal{A}) V_{h} \subseteq \operatorname{span}\left\{\phi_{i}: i \in \mathcal{I}_{h}^{\mathrm{nc}}\right\}=: \operatorname{span} \Phi_{h}^{\mathrm{nc}} .
$$

Then, setting $V_{i}^{\mathrm{nc}}:=\operatorname{span} \phi_{i}$, the collection

$$
\mathcal{S}=\left\{V_{i}^{\mathrm{nc}}: i \in \mathcal{I}_{h}^{\mathrm{nc}}\right\} \cup\left\{V_{i}^{\mathrm{c}}: i \in \mathcal{I}_{h}^{\mathrm{c}}\right\}
$$

is an energy stable splitting for $V_{h}$ in the sense of $(3.3)$.

Proof: In view of Theorem 4.4 it suffices to estimate the local contributions $\left\|\mid w^{K}\right\|_{h}, w^{K}=\chi_{K} w$, for $w \in V_{h}^{\text {nc }}$. More precisely, we shall prove that

$$
\sum_{K \in \mathcal{T}_{h}}\left\|w^{K}\right\|_{h}^{2} \lesssim \sum_{(K, p) \in \mathcal{I}_{h}^{\mathrm{nc}}}\left\|w_{K, p} \phi_{K, p}\right\|_{h}^{2} \lesssim \sum_{K \in \mathcal{T}_{h}}\left\|w^{K}\right\|_{h}^{2}
$$


holds with constants depending only on $\bar{k}$, the shape properties of $\mathcal{T}^{0}$, and the constants in 4.10 , , 2.3. Indeed writing

$$
w^{K}=\sum_{(K, p) \in \mathcal{I}_{h}^{\text {nc }}} w_{K, p} \phi_{K, p}
$$

we note from 4.28 and 4.29 that $\left\|\phi_{K, p}\right\|_{L_{\infty}(K)} \sim 1$. Hence, by 4.17 we have $\left\|w_{K, p} \phi_{K, p}\right\|_{h}^{2} \lesssim\left(C_{a, K}+\right.$ 1) $\left|w_{K, p}\right|^{2}$ with a further constant factor depending on $\bar{k}$ and the shape properties of $\mathcal{T}^{0}$. Using the fact that only a fixed finite number (depending on $\bar{k}$ ) of basis functions are supported on each $K$, and taking the normalization 4.29 into account, we conclude that

$$
\begin{aligned}
\left\|w^{K}\right\|\left\|_{h}^{2}\right\| w_{p}^{K}\|\|_{h}^{2} & \\
& \lesssim \sum_{(K, p) \in \mathcal{I}_{h}^{\mathrm{nc}}}\left\|w_{K, p} \phi_{K, p}\right\|_{h}^{2} \lesssim\left\|\left\{w_{K, p}\left\|\phi_{K, p}\right\|_{h}\right\}_{(K, p) \in \mathcal{I}_{h}^{\mathrm{nc}}}\right\|_{\ell_{\infty}}^{2} \\
& \lesssim\left\|\sum_{(K, p) \in \mathcal{I}_{h}^{\mathrm{nc}}} w_{K, p} \phi_{K, p}\right\|_{L_{\infty}(K)}^{2}=\|w\|_{L_{\infty}(K)}^{2},
\end{aligned}
$$

where the constants depend only on $\bar{k}$ as well as on $C_{a, K}, \gamma$ in 4.17 and 4.29 . Now we use again a scaling/norm equivalence argument and 4.10 to obtain

$$
\|w\|_{L_{\infty}(K)} \lesssim 2^{j(K)}\|w\|_{L_{2}(K)} \lesssim C^{*}\|w\|_{h(\omega(K))},
$$

where the remaining unspecified constant factor depends only on $\bar{k}$, and the shape properties of $\mathcal{T}^{0}$. Finally, we note again that for any edge $e \subset K^{\prime} \cap K^{\prime \prime}$ one has $\|[w]\|_{L_{2}(e)}^{2} \leq\left\|w^{K^{\prime}}\right\|_{L_{2}(e)}^{2}+\left\|w^{K^{\prime \prime}}\right\|_{L_{2}(e)}^{2}$, hence

$$
\|w\|_{h(\omega(K))}^{2} \leq \sum_{K^{\prime} \in \mathcal{T}_{h}: K^{\prime} \subset \omega(K)}\left\|w^{K^{\prime}}\right\|_{h^{2}}^{2}
$$

Combining now (4.34, 4.35, 4.36) and using again 4.11, yields 4.33) and completes the proof.

For the concrete realization of the preconditioned problem $(3.8)$, it now remains to identify viable choices of the auxiliary bilinear forms $b_{i}(\cdot, \cdot)$ for $i \in \mathcal{I}_{h}:=\mathcal{I}_{h}^{\mathrm{c}} \cup \mathcal{I}_{h}^{\text {nc }}$. Here $\mathcal{I}_{h}^{\mathrm{c}}, \mathcal{I}_{h}^{\text {nc }}$ are the index sets for stable splittings $\left\{V_{i}^{\mathrm{c}}\right\},\left\{V_{i}^{\mathrm{nc}}\right\}$ of $V_{h}^{\mathrm{c}}, V_{h}^{\mathrm{nc}}$, respectively, as discussed above. Likewise, the conforming splitting typically contains the full coarse grid space. In general, let $\omega(i)$ denote the union of all the supports of the nodal basis functions spanning the subspace $V_{i}^{\text {c }}$ (which could be all of $\Omega$ when $i$ refers to a full coarse level space).

Some admissible choices for the $b_{i}(\cdot, \cdot)$ are the following:

$$
\begin{cases}a(v, w)_{K}+\sum_{e \in \mathcal{E}_{h}, e \subset \partial K}|e|^{-1} \int_{e}[v][w] & \text { or } \\ |K|^{-1}(v, w)_{K}+\sum_{e \in \mathcal{E}_{h}, e \subset \partial K}|e|^{-1} \int_{e}[v][w] & \text { when } i=(K, p) \in \mathcal{I}_{h}^{\mathrm{nc}} \\ a(v, w)_{\omega(i)} & \text { or } \\ |\omega(i)|^{-1}(v, w)_{\omega(i)} & \text { when } i \in \mathcal{I}_{h}^{\mathrm{c}} .\end{cases}
$$

REMARK 4.5. In all the above cases in (4.37) there exist constants $c_{b}, C_{b}$ depending only on the degree $k$, the shape properties of $\mathcal{T}^{0}$ and possibly on the coefficients in the bilinear form $a(\cdot, \cdot)$, such that (3.4) holds.

Thus we can summarize these findings as follows.

TheOREm 4.6. Assume that $\mathcal{A}$ is admissible. Then the additive Schwarz preconditioner based on the the above splittings (for the respective realizations of $V_{h}^{\mathrm{c}}$, see Section 5.1.1) and for any of the bilinear forms from (4.37) is asymptotically optimal in the sense of Theorem 3.1 .

The robustness of the scheme with respect to the diffusion coefficients in $a(\cdot, \cdot)$ will be discussed in more detail elsewhere. 
5. Projecting onto the minimal conforming subspace. In principle the results of the previous section offer a general framework for a family of stable splittings and associated preconditioners. In this paper we are content with one specific realization of an admissible projector $\mathcal{A}$ and the identification of corresponding stable splittings for the conforming part. Specifically, we choose $\mathcal{A}=\mathcal{A}_{1}$ so as to map $V_{h}$ into continuous piecewise affine elements - in this sense the smallest possible subspace of $H_{0}^{1}$-conforming trial functions, i.e.

$$
V_{h, 1}^{\mathrm{c}}:=\mathcal{A}_{1} V_{h}=H_{0}^{1}(\Omega) \cap \mathbb{P}_{1}\left(\mathcal{T}_{h}\right) .
$$

In general it differs from $V_{h} \cap H_{0}^{1}(\Omega)$ whenever higher degrees are permitted.

An alternative would be to have $\mathcal{A}=\mathcal{A}_{k}$ produce globally continuous finite elements of the same degree as the original discontinuous elements and in that sense to split off the maximal conforming subspace in $V_{h}$, i.e.

$$
V_{h, k}^{\mathrm{c}}:=\mathcal{A}_{k} V_{h}=V_{h} \cap H_{0}^{1}(\Omega) .
$$

This will be elaborated on in a forthcoming paper.

In principle, such averaging operators are familiar in other contexts concerning DG discretizations, see e.g. [10]. The form of such projectors in the presence of hanging vertices is to our knowledge less familiar. Since hanging vertices do appear to add some complication we shall give in what follows an explicit selfcontained construction.

5.1. The construction of $\mathcal{A}_{1}$. The construction of $\mathcal{A}_{1}$ is inspired by the results in [10, 11] but has to cope with hanging nodes. For this reason, let us remember that we call regular those vertices that are not hanging. In particular, every vertex on the boundary $\partial \Omega$ is regular. Since boundary vertices are subject to a zero boundary condition in $H_{0}^{1}(\Omega)$, we can define $\mathcal{A}_{1} v$ by prescribing its nodal values at every interior regular vertex $n$, following

$$
\left(\mathcal{A}_{1} v\right)(n):=\frac{1}{\#\left(\mathcal{T}_{h}(n)\right)} \sum_{K \in \mathcal{T}_{h}(n)} v^{K}(n)
$$

Since $V_{h, 1}^{\mathrm{c}}$ is always a subset of $V_{h}$ (that is, for any degree distribution $k$ ), the space

$$
V_{h, 1}^{\mathrm{nc}}:=\left(I-\mathcal{A}_{1}\right) V_{h}
$$

stands as a possible candidate for complementing $V_{h, 1}^{\text {c }}$, i.e. we have

$$
V_{h}=V_{h, 1}^{\mathrm{c}} \oplus V_{h, 1}^{\mathrm{nc}} \text {. }
$$

5.1.1. Energy stable splittings for $V_{h, 1}^{\mathrm{c}}$. To describe an energy frame $\Phi_{h, 1}^{\mathrm{c}}$ for $V_{h, 1}^{\mathrm{c}}$ we can essentially resort to known results. In fact $\Phi_{h, 1}^{c}$ has multilevel structure and is comprised by all nodal continuous basis functions associated with all predecessors of $\mathcal{T}_{h}$ within the tree $\widehat{\mathcal{T}}_{h}$. To describe this, let us denote again by $\mathcal{T}^{j}$ the $j$ th uniform dyadic refinement of $\mathcal{T}^{0}$ and let

$$
\mathcal{T}_{h}^{j}:=\left\{K \in \mathcal{T}^{j}: \exists K^{\prime} \in \mathcal{T}_{h} \text { s.t. } K^{\prime} \subseteq K\right\}=\mathcal{T}^{j} \cap \widehat{\mathcal{T}}_{h},
$$

i.e. $\mathcal{T}_{h}^{j}$ is comprised of all level $j$ triangles that appear in the refinement history leading to $\mathcal{T}_{h}$. Moreover, let

$$
\Omega_{h}^{j}:=\bigcup\left\{K: K \in \mathcal{T}_{h}^{j}\right\}
$$

the area covered by the elements in $\mathcal{T}_{h}^{j}$. The subspace $V_{h, 1}^{\mathrm{c}}:=\mathcal{A}_{1} V_{h}$ consists of all continuous piecewise linear functions on $\mathcal{T}_{h}$. Consider now $\mathcal{N}_{h, 1}^{j, c}$ the set of those vertices that belong to a triangle in $\mathcal{T}_{h}^{j}$ and lie in the interior of $\Omega_{h}^{j}$, i.e.

$$
\mathcal{N}_{h, 1}^{j, \mathrm{c}}:=\bigcup\left\{\mathcal{N}_{1}(K) \backslash \partial \Omega_{h}^{j}: K \in \mathcal{T}_{h}^{j}\right\}
$$


Note that since hanging vertices are always located on some boundary $\partial \Omega_{h}^{j}$, the vertices in $\mathcal{N}_{h, 1}^{j, \mathrm{c}}$ are neither hanging, nor they lie in the boundary of the corresponding domains.

Now define the multilevel index set

$$
\mathcal{I}_{h, 1}^{\mathrm{c}}:=\bigcup\left\{i=(j, n): j=0, \ldots, j_{h}, n \in \mathcal{N}_{h, 1}^{j, \mathrm{c}}\right\},
$$

where $j_{h}$ is the maximal level appearing in $\mathcal{T}_{h}$. With each $i=(j, n) \in \mathcal{I}_{h, 1}^{\mathrm{c}}$ we associate now the standard nodal (piecewise affine) hat function $\varphi_{i}^{c}$ at the vertex $n$ supported on the star of triangles in $\mathcal{T}_{h}^{j}$ sharing $n$. Clearly $\varphi_{i}^{\mathrm{c}}$ is continuous, and it immediately follows from (4.17) that

$$
\left\|\mid \varphi_{i}^{\mathrm{c}}\right\|_{h}=a\left(\varphi_{i}^{\mathrm{c}}, \varphi_{i}^{\mathrm{c}}\right)^{1 / 2} \sim 1, \quad i \in \mathcal{I}_{h, 1}^{\mathrm{c}},
$$

holds with constants depending only on the constants in 2.3 and on the shape properties of $\mathcal{T}^{0}$. Now set

$$
\Phi_{h, 1}^{\mathrm{c}}:=\left\{\varphi_{i}^{\mathrm{c}}: i \in \mathcal{I}_{h, 1}^{\mathrm{c}}\right\} .
$$

It is easy to see that $\Phi_{h, 1}^{\mathrm{c}}$ spans $V_{h, 1}^{\mathrm{c}}$ and it is known (see e.g. [14, 7, 5]) that this collection is an energy-stable splitting, i.e.

$$
a(v, v) \sim \inf _{\substack{v_{i} \in \mathbb{R} \\ v=\sum_{i \in \mathcal{I}_{h, 1}^{\mathrm{c}}}}}\left\{\sum_{v_{i} \varphi_{i}^{\mathrm{c}}} \sum_{i \in \mathcal{I}_{h, 1}^{\mathrm{c}}} v_{i}^{2} a\left(\varphi_{i}^{\mathrm{c}}, \varphi_{i}^{\mathrm{c}}\right)\right\}, \quad v \in V_{h, 1}^{\mathrm{c}},
$$

where the constants depend only on $\mathcal{T}^{0}$ and the constants $c_{a}, C_{a}$

5.1.2. The Jackson estimate for $\mathcal{A}_{1}$. We shall confirm now the validity of Property 4.3 for $\mathcal{A}=\mathcal{A}_{1}$. To this end, let us first establish the following intermediate result that is only valid for piecewise affine functions. Remember that the different sets $\mathcal{E}_{h}(n), \mathcal{N}_{h, 1}^{*}(n), \mathcal{T}_{h}(n), \ldots$ which denote local neighborhoods made of mesh elements, are defined in Section 4.2

Lemma 5.1. If $v \in \mathbb{P}_{1}\left(\mathcal{T}_{h}\right)$ is a piecewise affine function, then we have for any node $n \in \mathcal{N}_{h, 1}$

$$
\left|\left(v-\mathcal{A}_{1} v\right)^{K}(n)\right| \leq \sum_{n^{\prime} \in \mathcal{N}_{h, 1}^{*}(n)} \sum_{e \in \mathcal{E}_{h}\left(n^{\prime}\right)}\|[v]\|_{L_{\infty}(e)}, \quad \forall K \in \mathcal{T}_{h} .
$$

Proof: We begin with the following basic estimate, independent of the operator $\mathcal{A}_{1}$ : at any vertex $n \in \mathcal{N}_{h, 1}$, we have

$$
\left|\left(v^{K}-v^{K^{\prime}}\right)(n)\right| \leq \sum_{e \in \mathcal{E}_{h}(n)}\|[v]\|_{L_{\infty}(e)}, \quad K, K^{\prime} \in \mathcal{T}_{h}(n)
$$

for any $v \in V_{h}$. Indeed, since $K$ and $K^{\prime}$ do not necessarily share an edge, write $\mathcal{T}_{h}(n)=\left\{K_{1}, \ldots, K_{M}\right\}$ the (closed) triangles containing $n$, in such a way that $K_{i}$ and $K_{i+1}$ have the edge $e_{i} \in \mathcal{E}_{h}(n)$ as their intersection, and assume that $K=K_{M}$ and $K^{\prime}=K_{m}$ with $m<M$ (observe from the definition of the set $\mathcal{E}_{h}$ that this is possible even when $n$ is a hanging vertex). It is then easily seen that

$$
\left|\left(v^{K}-v^{K^{\prime}}\right)(n)\right| \leq \sum_{i=m}^{M-1}\left|\left(v^{K_{i+1}}-v^{K_{i}}\right)(n)\right| \leq \sum_{i=m}^{M-1}\|[v]\|_{L_{\infty}\left(e_{i}\right)},
$$

which clearly gives $(5.10)$.

Actually, because the jumps over boundary edges are defined as if $v$ was extended to 0 outside $\Omega$, this argument yields the following estimate when $n$ is on $\partial \Omega$

$$
\left|v^{K}(n)\right| \leq \sum_{e \in \mathcal{E}_{h}(n)}\|[v]\|_{L_{\infty}(e)}, \quad n \in \partial \Omega, K \in \mathcal{T}_{h}(n) .
$$


Now to prove the assertion of the lemma we consider $n \in K$ and distinguish three cases: (i) $n$ is on the boundary $\partial \Omega$, (ii) $n$ is a regular vertex in the interior of $\Omega$, and finally (iii) $n$ is hanging, i.e. there is (only) one triangle that contains $n$ and for which $n$ is not a vertex. Note that in the two first cases we have $\mathcal{N}_{h, 1}^{*}(n)=\{n\}$.

In the case (i), 5.11) readily yields Lemma 5.1 since then we have $\mathcal{A}_{1} v=0$.

In the case (ii) where $n$ is interior and regular, by definition $(5.3)$ of $\mathcal{A}_{1}$, we have

$$
\left|\left(v-\mathcal{A}_{1} v\right)^{K}(n)\right| \leq \frac{1}{\#\left(\mathcal{T}_{h}(n)\right)} \sum_{K^{\prime} \in \mathcal{T}_{h}(n)}\left|\left(v^{K}-v^{K^{\prime}}\right)(n)\right|,
$$

so that Lemma 5.1 now follows from 5.10 .

Finally, in the case (iii) where $n$ is hanging, denote by $K^{\prime}$ the unique triangle in $\mathcal{T}_{h}(n)$ for which $n$ is not a vertex, and by $\left[n^{\prime}, n^{\prime \prime}\right]$ the edge of $K^{\prime}$ for which $n$ is the midpoint. Since both $v$ and $\mathcal{A}_{1} v$ are affine on $K^{\prime}$, we have

$$
\left|\left(v-\mathcal{A}_{1} v\right)^{K^{\prime}}(n)\right| \leq \frac{1}{2}\left(\left|\left(v-\mathcal{A}_{1} v\right)^{K^{\prime}}\left(n^{\prime}\right)\right|+\left|\left(v-\mathcal{A}_{1} v\right)^{K^{\prime}}\left(n^{\prime \prime}\right)\right|\right) .
$$

Now from the grading of $\mathcal{T}_{h}$, we know that $n^{\prime}$ and $n^{\prime \prime}$ are never hanging themselves, so that 5.12 applies, providing, on account of 5.10 ,

$$
\left|\left(v-\mathcal{A}_{1} v\right)^{K^{\prime}}(\check{n})\right| \leq \sum_{e \in \mathcal{E}_{h}(\check{n})}\|[v]\|_{L_{\infty}(e)}, \quad \check{n}=n^{\prime} \text { or } n^{\prime \prime} .
$$

Writing then

$$
\left|\left(v-\mathcal{A}_{1} v\right)^{K}(n)\right| \leq\left|v^{K}(n)-v^{K^{\prime}}(n)\right|+\left|\left(v-\mathcal{A}_{1} v\right)^{K^{\prime}}(n)\right|,
$$

we prove 5.9$)$ in the case (iii) by combining the estimates 5.13 , (5.10) and (5.14), since then we have by definition $\mathcal{N}_{h, 1}^{*}(n)=\left\{n, n^{\prime}, n^{\prime \prime}\right\}$, see 4.4.

We are now ready to verify the validity of Property 4.3 for $\mathcal{A}_{1}$.

Proposition 5.2. The projector $\mathcal{A}_{1}$ has Property 4.3 where $C^{*}$ in 4.10 depends only on the shape properties of $\mathcal{T}^{0}$, on $\bar{k}$, and on $\max _{K^{\prime} \subset \omega(K)} \underline{\lambda}_{K^{\prime}}^{-1}$.

Proof: To estimate the projection error $\left(I-\mathcal{A}_{1}\right) v$ consider the interpolation operator $P_{1}: V_{h} \rightarrow \mathbb{P}_{1}\left(\mathcal{T}_{h}\right)$ given by

$$
\left\{P_{1} v\right\}^{K}(n):=v^{K}(n), \quad K \in \mathcal{T}_{h}, n \in \mathcal{N}_{1}(K),
$$

for which the estimate

$$
\left\|\left(I-P_{1}\right) v\right\|_{L_{2}(K)} \lesssim 2^{-j(K)}\|\nabla v\|_{L_{2}(K)}
$$

is well known with a constant depending only on the shape properties of $\mathcal{T}^{0}$. In view of $[5.3]$, it is readily seen that $\mathcal{A}_{1} P_{1}=\mathcal{A}_{1}$, so that the projection error reads

$$
\left(I-\mathcal{A}_{1}\right) v=\left(I-P_{1}\right) v+\left(I-\mathcal{A}_{1}\right) P_{1} v .
$$

Writing $w:=\left(I-\mathcal{A}_{1}\right) P_{1} v \in \mathbb{P}_{1}\left(\mathcal{T}_{h}\right)$ it follows that

$$
\left\|\left(I-\mathcal{A}_{1}\right) v\right\|_{L_{2}(K)} \lesssim 2^{-j(K)}\|\nabla v\|_{L_{2}(K)}+\|w\|_{L_{2}(K)}
$$

Now, denote by $\left\{\phi_{(K, n)}^{1}: n \in \mathcal{N}_{1}(K)\right\}$ the discontinuous nodal basis of $\mathbb{P}_{1}(K)$. On any triangle $K \in \mathcal{T}_{h}$ we have

$$
\|w\|_{L_{2}(K)} \leq \sum_{n \in \mathcal{N}_{1}(K)}\left|w^{K}(n)\right|\left\|\phi_{(K, n)}^{1}\right\|_{L_{2}} \lesssim \sum_{n \in \mathcal{N}_{1}(K)} 2^{-j(K)}\left|w^{K}(n)\right|
$$


Applying then Lemma 5.1 to $P_{1} v$, and recalling that the set $\mathcal{E}_{h}^{*}(K)$ is given by (4.6), we find

$$
\|w\|_{L_{2}(K)} \lesssim 2^{-j(K)} \sum_{e \in \mathcal{E}_{h}^{*}(K)}\left\|\left[P_{1} v\right]\right\|_{L_{\infty}(e)},
$$

where the constant depends only on the shape properties of $\mathcal{T}^{0}$.

Now assume for the moment that for any edge $e \in \mathcal{E}_{h}$ there is one triangle $K_{e}$ for which $e \subset \partial K_{e}$, and such that the inequality

$$
\left\|\left[P_{1} v\right]\right\|_{L_{\infty}(e)} \lesssim\|[v]\|_{L_{\infty}(e)}+\|\nabla v\|_{L_{2}\left(K_{e}\right)}
$$

holds with a constant that only depends on the shape properties of $\mathcal{T}^{0}$. Since for every $e$ in $\mathcal{E}_{h}^{*}(K)$, any such $K_{e}$ is in $\mathcal{T}_{h}^{*}(K)$, see 4.6) and 4.7), this would yield

$$
\|w\|_{L_{2}(K)} \lesssim 2^{-j(K)}\left(\sum_{e \in \mathcal{E}_{h}^{*}(K)}\|[v]\|_{L_{\infty}(e)}+\sum_{K^{\prime} \in \mathcal{T}_{h}^{*}(K)}\|\nabla v\|_{L_{2}\left(K^{\prime}\right)}\right) .
$$

Now since $[v]$ is a polynomial on any edge $e \in \mathcal{E}_{h}$, we recall from 4.17 that $\|[v]\|_{L_{\infty}(e)} \sim|e|^{-1 / 2}\|[v]\|_{L_{2}(e)}$. Moreover, as in 2.6 each term $\|\nabla v\|_{L_{2}\left(K^{\prime}\right)}$ can be bounded by $C \underline{\lambda}_{K^{\prime}}^{-1} a(v, v)_{K^{\prime}}$ where $C$ depends only on $\bar{k}$ and the shape properties of $\mathcal{T}^{0}$. Note that this means that, aside from the dependence on $k$ and the shape properties of $\mathcal{T}^{0}$, the constant is of the order

$$
C \sim \max _{e \subset \omega(K)} \gamma_{e}
$$

see the remarks following 2.7 .

Since the sets $\mathcal{E}_{h}^{*}(K)$ are of uniformly bounded cardinality (depending only on $\mathcal{T}^{0}$ ), we find (upon using also (5.17) and 4.9) that

$$
\left\|\left(I-\mathcal{A}_{1}\right) v\right\|_{L_{2}(K)} \lesssim C^{*} 2^{-j(K)}\|v\|_{h(\omega(K))},
$$

where the constant $C^{*}$ is of the form $C \max _{K^{\prime} \subset \omega(K)} \underline{\lambda}_{K^{\prime}}^{-1}, C$ depending only on $\bar{k}$ and the shape properties of $\mathcal{T}^{0}$. This completes the proof of Lemma 4.3 .

Thus, it only remains to prove (5.18). Here three situations may occur: either (1) the edge $e$ is on the boundary $\partial \Omega$, or this is not the case and we can write $e=K_{e} \cap K_{e}^{\prime}$ with $K_{e}, K_{e}^{\prime} \in \mathcal{T}_{h}$. Now either (2) $e$ is an edge of both $K_{e}$ and $K_{e}^{\prime}$, or again (3) this is not not the case. In the two first cases, we observe that $\left[P_{1} v\right]_{e}=P_{1}\left([v]_{e}\right)$, so that

$$
\left\|\left[P_{1} v\right]\right\|_{L_{\infty}(e)} \leq\|[v]\|_{L_{\infty}(e)}
$$

is obvious. Now according to the structure of graded triangulations, the only possible configuration in case (3) is represented in Figure 4.1 with $e=\left[n^{\prime}, n\right]$. Setting then $K_{e}$ and $K_{e}^{\prime}$ to be the coarser and finer triangles, respectively (i.e. $K$ and $K^{\prime}$ in Figure 4.1), we observe that $P_{1} v$ is affine on $K_{e}$. Therefore

$$
\left\|\left[P_{1} v\right]\right\|_{L_{\infty}(e)}=\max \left\{\left|v^{K_{e}}\left(n^{\prime}\right)-v^{K_{e}^{\prime}}\left(n^{\prime}\right)\right|,\left|\frac{1}{2}\left(v^{K_{e}}\left(n^{\prime}\right)+v^{K_{e}}\left(n^{\prime \prime}\right)\right)-v^{K_{e}^{\prime}}(n)\right|\right\} .
$$

Writing

$$
\frac{1}{2}\left(v^{K_{e}}\left(n^{\prime}\right)+v^{K_{e}}\left(n^{\prime \prime}\right)\right)=\frac{1}{2}\left(v^{K_{e}}\left(n^{\prime}\right)-v^{K_{e}}(n)\right)+\frac{1}{2}\left(v^{K_{e}}(n)-v^{K_{e}}\left(n^{\prime \prime}\right)\right)+v^{K_{e}}(n),
$$

we find (with an absolute constant)

$$
\left\|\left[P_{1} v\right]\right\|_{L_{\infty}(e)} \lesssim\|[v]\|_{L_{\infty}(e)}+\left|v^{K_{e}}\left(n^{\prime}\right)-v^{K_{e}}(n)\right|+\left|v^{K_{e}}(n)-v^{K_{e}}\left(n^{\prime \prime}\right)\right| .
$$

Using a scaling argument together with the fact that $v$ is polynomial on $K_{e}$, one finally verifies that

$$
\left|v^{K_{e}}(n)-v^{K_{e}}(\check{n})\right| \lesssim\|\nabla v\|_{L_{2}\left(K_{e}\right)}, \quad \check{n}=n^{\prime} \text { or } n^{\prime \prime},
$$




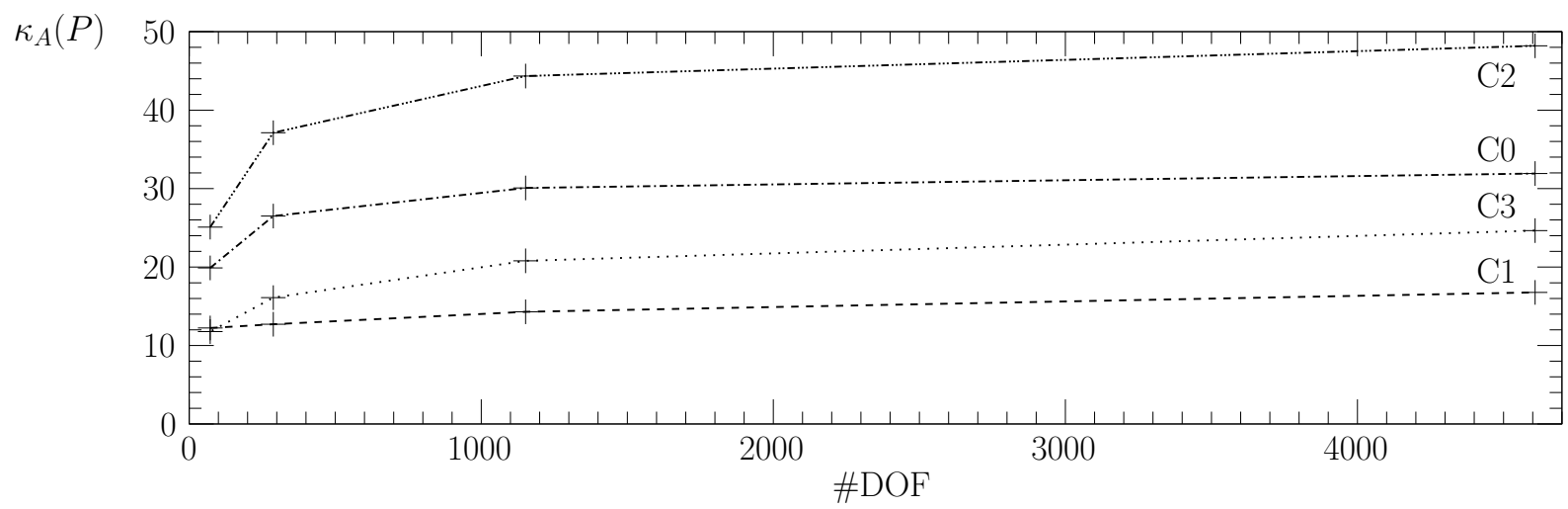

FIG. 6.1. Condition numbers for the cases C0, C1, C2 and C3 for $\gamma=10$ and $d_{i}=1$.

holds with a constant that depends only on the shape properties of $\mathcal{T}^{0}$. Collecting the above estimates, one confirms (5.18) and completes the proof.

Combining Corollary 4.5 (5.8) and Propositions 5.2, yields the following result.

Corollary 5.3. Let $\mathcal{I}_{h, 1}^{\mathrm{c}}$ and $\varphi_{i}^{\mathrm{c}}$ be defined as in Section 5.1.1. Then for any basis $\left\{\phi_{K, p}: K \in\right.$ $\left.\mathcal{T}_{h}, p \in \mathcal{N}_{k}(K)\right\}$ of $V_{h}$ constructed as in 4.28) (such as a piecewise Lagrange basis), the collection

$$
\mathcal{S}_{1}:=\left\{\operatorname{span}\left(\varphi_{i}^{\mathrm{c}}\right): i \in \mathcal{I}_{h, 1}^{\mathrm{c}}\right\} \cup\left\{\operatorname{span}\left(\phi_{K, p}\right): K \in \mathcal{T}_{h}, p \in \mathcal{N}_{k}(K)\right\}
$$

is a stable splitting of $V_{h}$ in the sense of (3.3). In particular, the corresponding problem $(3.8)$ is equivalent to the original equation (3.2) and has a condition number bounded by a constant independent of $\mathcal{T}_{h}$.

REMARK 5.1 (proof of Theorem 1.1). Theorem 1.1 is an immediate consequence of Corollary 4.5 and Proposition 5.2, observing that $V_{h}^{c}$ can always be regarded as a splitting of itself.

6. Numerical Experiments. We now present some first numerical experiments for the simple model problem $a(u, v)=\int_{\Omega} \nabla u \cdot \nabla v$ where $\Omega$ is an L-shaped domain in $\mathbb{R}^{2}$ obtained by cutting out the upper right square $(1,2]^{2}$ from $[0,2]^{2}$. Moreover, we shall only consider the first degree case $\bar{k}=1$ on a uniformly refined grid. At this point we are mainly interested in the quantitative influence of the specific choice of the auxiliary bilinear forms $b_{i}(\cdot, \cdot)$, of the stabilization parameter $\gamma$, and of the low dimensional subspaces $V_{i}$ in the nonconforming part.

We shall use the following notation: $d_{i}:=\operatorname{dim} V_{i}$ denotes the dimension of the subspaces in the nonconforming splitting. $d_{i}=3$ refers to the case that $V_{i}$ is spanned by the three nodal basis functions on each triangle in $\mathcal{T}_{h}$.

The case $\mathrm{C} 0$ refers to the choice

$$
b_{i}(v, w)= \begin{cases}a(v, w)_{K}+\sum_{e \in \mathcal{E}_{h}, e \in \partial K}|e|^{-1} \int_{e}[v][w] & \text { for } \quad i=(K, p) \in \mathcal{I}_{h}^{\mathrm{nc}} \\ a(v, w)_{\omega(i)} & \text { for } \quad i=(j, p) \in \mathcal{I}_{h}^{\mathrm{c}},\end{cases}
$$

of the auxiliary inner products (see 4.37), while $\mathrm{C} 1$ corresponds to

$$
b_{i}(v, w)=\left\{\begin{array}{lll}
|K|^{-1}(v, w)_{K}+\sum_{e \in \mathcal{E}_{h}, e \in \partial K}|e|^{-1} \int_{e}[v][w] & \text { for } & i=(K, p) \in \mathcal{I}_{h}^{\mathrm{nc}}, \\
2^{2 j}(v, w)_{\omega(i)} & \text { for } \quad i=(j, p) \in \mathcal{I}_{h}^{\mathrm{c}} .
\end{array}\right.
$$

In order to test the preconditioning effect we have estimated the condition numbers using power iteration. Figure 6.1 shows the dependence of the condition number of the preconditioned operator $\kappa\left(P_{h}\right)$ on the number of degrees of freedom for four selections of $b_{i}(\cdot, \cdot)$. In addition to $\mathrm{C} 0$ and $\mathrm{C} 1$, we consider the cases $\mathrm{C} 2$ and $\mathrm{C} 3$ which are given by combining the first line of $(6.1)$ with the second line of $(6.2)$ and vice versa, respectively. Qualitatively for all choices of $b_{i}(\cdot, \cdot)$ the condition numbers of the preconditioned operator $P_{h}$ show the same bounded behavior.

Recall that $\gamma=10$ is usually considered as an appropriate choice. We consider first the moderate values $\gamma=5, \gamma=7.5$ in the case $d_{i}=1$. 


\begin{tabular}{|r|r|r|rrrrr|}
\hline \multirow{2}{*}{ Level } & \multirow{2}{*}{ \#DOF } & \multicolumn{5}{|c|}{$\kappa$ for preconditioner (case/ $\gamma$ ) } \\
& & \multicolumn{1}{|c|}{ none } & $\mathrm{C} 0 / 5$ & $\mathrm{C} 1 / 5$ & $\mathrm{C} 0 / 7.5$ & $\mathrm{C} 1 / 7.5$ \\
\hline 1 & 72 & 13.62 & 12.24 & 13.62 & 15.60 & 11.24 \\
2 & 288 & 106.84 & 14.24 & 20.45 & 20.22 & 13.60 \\
3 & 1152 & 414.51 & 15.53 & 26.19 & 22.72 & 17.31 \\
4 & 4608 & 1646.80 & 16.23 & 30.94 & 24.03 & 20.39 \\
\hline
\end{tabular}

For small $\gamma$ the choice $\mathrm{C} 0$ appears to be slightly superior while for larger $\gamma$ roles reverse, as shown in the next table for $\gamma=10, \gamma=15$.

\begin{tabular}{|r|r|r|rrrrr|}
\hline \multirow{2}{*}{ Level } & \multirow{2}{*}{ \#DOF } & \multicolumn{5}{|c|}{$\kappa$ for preconditioner (case $/ \gamma$ ) } \\
& & none & C0 $/ 10$ & C1/10 & C0/15 & C1/15 \\
\hline 1 & 72 & 12.20 & 19.89 & 12.25 & 28.56 & 13.95 \\
2 & 288 & 210.10 & 26.50 & 12.71 & 39.09 & 14.53 \\
3 & 1152 & 825.76 & 30.07 & 14.30 & 44.79 & 14.71 \\
4 & 4608 & 3294.20 & 31.92 & 16.76 & 47.74 & 14.77 \\
\hline
\end{tabular}

We turn now to the case $d_{i}=3$ for $\gamma=5, \gamma=7.5$.

\begin{tabular}{|r|r|r|r|r|r|r|}
\hline \multirow{2}{*}{ Level } & \multirow{2}{*}{ \#DOF } & \multicolumn{5}{|c|}{$\kappa$ for preconditioner $($ case $/ \gamma)$} \\
& & none & $\mathrm{C} 0 / 5$ & $\mathrm{C} 1 / 5$ & $\mathrm{C} 0 / 7.5$ & $\mathrm{C} 1 / 7.5$ \\
\hline 1 & 72 & 13.62 & 12.24 & 13.62 & 15.60 & 11.11 \\
2 & 288 & 106.84 & 14.24 & 20.45 & 20.22 & 13.60 \\
3 & 1152 & 414.51 & 15.53 & 26.19 & 22.72 & 17.31 \\
4 & 4608 & 1646.80 & 16.23 & 30.92 & 24.03 & 20.38 \\
\hline
\end{tabular}

and further again for $\gamma=10, \gamma=15$.

\begin{tabular}{|r|r|r|rrrrr|}
\hline \multirow{2}{*}{ Level } & \multirow{2}{*}{ \#DOF } & \multicolumn{5}{|c|}{$\kappa$ for preconditioner (case $/ \gamma)$} \\
& & \multicolumn{1}{|c|}{ none } & $\mathrm{C} 0 / 10$ & $\mathrm{C} 1 / 10$ & $\mathrm{C} 0 / 15$ & $\mathrm{C} 1 / 15$ \\
\hline 1 & 72 & 12.20 & 23.45 & 8.19 & 33.89 & 9.30 \\
2 & 288 & 210.10 & 32.67 & 10.59 & 48.19 & 9.21 \\
3 & 1152 & 825.76 & 37.96 & 13.48 & 56.50 & 10.08 \\
4 & 4608 & 3294.20 & 40.72 & 15.88 & 60.85 & 11.83 \\
\hline
\end{tabular}

which shows a similar effect as for $d_{i}=1$ before.

We conclude with inspecting the number of cg-iterations for significantly larger problem sizes to reduce the relative error, that is the quotient of the $l_{2}$-norms of residual and right hand side vectors, to $10^{-5}$. In this example, we have chosen $\gamma=10, f=1$ on $\Omega$ as right hand side and zero as the initial guess for the conjugate gradient method. The results in the following table show that the number of iterations remains indeed bounded independently of the problem size.

\begin{tabular}{|r|r|r|rrrr|}
\hline \multirow{2}{*}{ Level } & \multirow{2}{*}{ \#DOF } & \multicolumn{5}{|c|}{ Number of iterations } \\
& & none & C0 $/ 1$ & C0 $/ 3$ & C1/1 & C1/3 \\
\hline 1 & 72 & 25 & 20 & 21 & 17 & 14 \\
2 & 288 & 56 & 25 & 27 & 17 & 16 \\
3 & 1152 & 107 & 27 & 30 & 16 & 16 \\
4 & 4608 & 212 & 28 & 31 & 16 & 16 \\
5 & 18432 & 426 & 28 & 31 & 17 & 16 \\
6 & 73728 & 861 & 27 & 31 & 17 & 17 \\
7 & 294912 & 1742 & 27 & 30 & 17 & 17 \\
8 & 1179648 & 3558 & 26 & 29 & 18 & 17 \\
9 & 4718592 & 7226 & 26 & 29 & 18 & 18 \\
\hline
\end{tabular}


7. Concluding Remarks. We have reduced the problem of devising multilevel Schwarz preconditioners for the Symmetric Interior penalty DG formulation to the availability of admissible averaging projectors provided that the underlying meshes satisfy a certain grading property. We have specified one such projector for which uniformly bounded condition numbers are proved under no extra regularity conditions for any graded triangulations and fixed highest polynomial degree. First numerical experiments illustrate the theoretical findings for a simple model problem and piecewise linear trial functions. Alternative splittings for higher order discretizations will be explored in a forthcoming paper together with more extensive numerical experiments for variable coefficients.

\section{REFERENCES}

[1] D. N. ARnold, An interior penalty finite element method with discontinuous elements, SIAM J. Numer. Anal., 19 (1982), pp. $742-760$.

[2] D. N. Arnold, F. Brezzi, B. Cockburn, And L. D. Marini, Unified analysis of discontinuous Galerkin methods for elliptic problems, SIAM J. Numer. Anal., 39 (2002), pp. 1749-1779.

[3] J. H. Bramble, J. E. Pasciak, and J. Xu, Parallel multilevel preconditioners, Math. Comp., 55 (1990), pp. 1-22.

[4] W. Dahmen, Adaptive approximation by multivariate smooth splines, J. Approx. Theory, 36 (1982), pp. 119-140.

[5] W. Dahmen, Wavelet and Multiscale Methods for Operator Equations, Acta Numerica, Cambridge University Press, 6 (1997), pp. 55-228.

[6] W. Dahmen and A. Kunoth, Multilevel Preconditioning, Numer. Math., 63 (1992), pp. 315-344.

[7] M. GRIEBel And P. Oswald, On the abstract theory of additive and multiplicative Schwarz algorithms, Numer. Math., 70 (1995), pp. 163-180.

[8] J. Gopalakrishnan and G. Kanschat, A multilevel discontinuous Galerkin method, Numer. Math., 95 (2003), pp. $527-550$.

[9] G. Farin, Curves and Surfaces for Computer Aided Geometric Design: A Practical Guide, Academic Press, New York, 1988.

[10] P. Houston, D. Schötzau, And T.P. WinleR, Energy norm a-posteriori error estimation of hp-adaptive discontinuous Galerkin methods for elliptic problems, Math. Models Methods Appl. Sci., 17 (2007), pp. 33-62.

[11] O. Karakashian And F. Pascal, A posteriori error estimates for a discontinuous Galerkin approximation of secondorder elliptic problems, SIAM J. Numer. Anal., 41 (2003), pp. 2374-2399.

[12] E. SüLI, C. Schwab, AND P. Houston, hp-DGFEM for partial differential equations with nonnegative characteristic form, in Discontinuous Galerkin Methods: Theory, Computation and Applications, B. Cockburn, G. E. Karniadakis, and C.-W. Shu, eds., Lecture Notes in Computational Science and Engineering, Springer, Berlin, 11 (2000), pp. 211-230.

[13] T.P. Wihler, P. Frauenfelder, And C. Schwab, Exponential convergence of the hp-DGFEM for diffusion problems, Comput. Math. Appl., 46 (2003), pp. 183-205.

[14] P. Oswald, Multilevel Finite Element Approximation - Theory and Applications, Teubner Skripten zur Numerik, B.G. Teubner, Stuttgart, 1994. 